\title{
10 Handlungen der verbalen Gewalt
}

„ihr Schmarotzer, [...] ihr verbrecherisches Ungeziefer, als Futter sollte man euch verarbeiten, damit ihr einmal nützlich seit. wir hassen Euch Judenschweine!“

[IBD_01.08.2006_ano_026]

Die Sprache kann, wie wir bereits an anderen Stellen erörtert haben, als Instrument eingesetzt werden, um Menschen mental zu verletzen, ihnen kognitiv und emotional Schaden zuzufügen, ihnen seelisches Leid anzutun und sie in Furcht und Sorge zu versetzen. Dass Wörter wie Waffen eingesetzt werden können, wird besonders offenkundig, wenn Äußerungen semantisch pejorativ und pragmatisch destruktiv ausgerichtet sind. Destruktiv bedeutet, dass der jeweilige Handlungswert einer Äußerung darin besteht, dem Adressaten durch den Vollzug des Sprechaktes etwas Negatives anzutun bzw. dieses anzukündigen oder zu wünschen. Die (kommunikative Funktion der) als Imperativ formulierte(n) Aufforderung von (1) zeigt diese destruktive Dimension und macht zugleich das hohe Aggressivitätspotenzial des Produzenten transparent:

$$
\text { „Verreckt!!!!“ [ZJD_10.01.2009_Her_001] }
$$

Viele judenfeindliche Äußerungen sind von ihrem Handlungswert her nicht nur repräsentativ und expressiv, geben also diffamierende Feststellungen (von deren Wahrhaftigkeit der Produzent überzeugt ist) und affektive Einstellungen wider wie in (2) und (3):

„Ich HASSE alle Israelis dieses Völkermordende Volk“ [IBD_16.01.2009_Kös_001]

3) „Ich spucke vor Abscheu vor ihnen auf den Boden!“ [ZJD_Gaza2009_632/816_Jun_001]

Vielmehr weist eine große Anzahl der Sprachhandlungen einen stark direktiven Charakter auf. Direktiva (Appellativa) zeichnen sich als verbale Aktivitäten dadurch aus, dass sie etwas Spezifisches beim Adressaten bewirken sollen. Meist soll der Rezipient zu einer Handlung bewegt werden, die außersprachliche Welt also mit dem Inhalt der Äußerung (und damit dem Wunsch des Produzenten nachkommend) in Übereinstimmung gebracht werden. Die intendierte Reaktion einer Aufforderung ist es z. B., auf Seiten des Rezipienten eine spezifische Aktivität in Gang zu setzen. 
(4) „Verlassen Sie das Land der Palästinenser!“ [IBD_22.03.2004_Sch_001]

(5) „Ich fordere Sie dazu auf, sich dafür einzusetzen, dass von Israel ausgehenden Zerstörungen eingestellt werden.“

[ZJD_Gaza2009_36/816_Fed_001]

Wie an (4) und (5) zu sehen ist, sind die meisten Sprechakte nie nur auf eine kommunikative Funktion festlegbar. Mit der Aufforderung in (4) vollzieht der Verfasser zugleich die Einstellungsbekundung, dass Israel unrechtmäßig Land okkupiere und somit keine Existenzberechtigung im Nahen Osten habe. Die direktive Sprachhandlung in (5) enthält neben der spezifischen Handlungsaufforderung zugleich implizit die Anschuldigung, die Zerstörungen würden allein von Israel ausgehen, und zudem die Unterstellung, der Zentralrat der Juden in Deutschland setze sich nicht gegen Gewalt im Nahen Osten ein. Dass die Aufforderung überhaupt an den Zentralrat und nicht an die Botschaft gesendet wurde, belegt zugleich die vom Sprecher als Fakt angenommene Verbindung zwischen deutschen Juden und israelischen Belangen. Dem Zentralrat wird so indirekt eine Mitverantwortung für israelische Militärhandlungen gegeben.

\subsection{Beschimpfen/Beleidigen, Drohen, Verwünschen}

In unserem Korpus dominieren drei Typen von besonders aggressiven Sprachhandlungen mit Appellfunktion: das Beschimpfen/Beleidigen, das Drohen ${ }^{1}$ und das Verwünschen von Juden. Diese Aggressivität spiegelt sich bereits vielfach in den Betreffzeilen der E-Mails wider: Meist wird bereits in dieser für den Adressaten zuerst wahrnehmbaren Informationszeile das Anliegen als verbaler Angriff formuliert, wie die folgenden Beispiele (die alle Betreffzeilen von E-Mails an den Zentralrat darstellen) exemplarisch zeigen:

$$
\text { „Betreff: Jüdische Verbrechen in Hebron“ [ZJD_05.12.2008_ano_001] }
$$

1 Drohen fällt nach der sprechakttheoretischen Einteilung in die Klasse der sogenannten Kommissiva. Bei diesen Sprachhandlungen verpflichtet sich der Produzent der Äußerung, selbst etwas in der Zukunft zu tun oder zu unterlassen, was negativ für den Rezipienten ist. Bei einer Drohung ist aber auch die direktive Handlungsdimension vorhanden, da der Rezipient mittels der Drohung entweder mental eingeschüchtert oder zu einer Handlung bewegt werden soll. Beschimpfen ist primär expressiv, d. h. der Produzent artikuliert seine Gefühle in Bezug auf den Rezipienten, will dadurch aber direktiv ausgerichtet erreichen, dass der Adressat gekränkt, verunsichert oder verängstigt wird. Da Beschimpfen und Drohen zu den aggressiven Sprechakten gehören, subsumieren wir diese Typen hier entsprechend. 

ren“[ZJD_Gaza2009_92/816_ano_001]

Wesentlich für das Beschimpfen ${ }^{2}$ ist die negative Adressatenbewertung: Der Angesprochene wird mittels pejorativer Wörter abgewertet. Damit verleiht der Produzent seiner stark affektiv geprägten Einstellung Ausdruck. Die intendierte Reaktion auf eine Beschimpfung ist das Beleidigt-Sein auf der Seite des Rezipienten. Die Bedeutung der gewählten Wörter ist bei Beschimpfungen entscheidend: Entsprechend kommt der pejorativen Lexik ein besonderer Status zu. Schimpfwörter zeichnen sich dadurch aus, dass sie etwas genuin Negatives im Benennungsakt auf den Adressaten prädizieren. Diese herabsetzende Referenzialisierung kann mittels Dehumanisierung (z. B. durch Tierbezeichnungen) oder Delegitimierung (z. B. Aberkennung von kognitiven Fähigkeiten wie bei Idiot) geschehen. Durch die Fokussierung auf als minderwertig erachtete geistige oder körperliche Merkmale wird das kommunikative Gegenüber als Person referenziell auf negative Merkmale reduziert, dadurch entsteht eine Inkongruenz zwischen Bedeutungsund Weltebene.

Pejorative Lexik, deren Funktion primär der Beschimpfung und Beleidigung des Angesprochenen dient, ist im mentalen Lexikon der Sprachbenutzer ${ }^{3}$ gespeichert und ihre Verwendung kann als usuell eingestuft werden. Viele antisemitische Schimpfwörter haben eine sehr lange Tradition (s. Kap.4) und werden teils seit Jahrhunderten benutzt, um Juden zu diskreditieren. Die folgenden Typen, ${ }^{4}$ die im aktuellen Sprachgebrauch zu beobachten sind, lassen sich unterscheiden: a. Tierbezeichnungen wie Schwein, Ratte, Aasgeier, Vieh. Hierzu zählen auch Lexeme aus dem mikrobiologischen Bereich wie Parasiten, Bazillen sowie Kreaturen, Brut.

2 Beschimpfen unterscheidet sich vom Schimpfen dadurch, dass es personenausgerichtet ist, während Schimpfen auch objekt- und situationsorientiert sein kann (vgl. So eine Mistlage, saumäßiges Verhalten etc.).

3 Bestimmte Lexeme ergeben aber erst in einem spezifischen Kontext eine pejorative Lesart. Jude ist die Bezeichnung für einen Angehörigen einer bestimmten Religionsgemeinschaft. Wird dieses Wort jedoch kontextuell erkennbar abwertend benutzt, wird es zu einem Schimpfwort (s. hierzu die Beispiele (30) bis (36)).

4 Wir konzentrieren uns hier auf die im judenfeindlichen Sprachgebrauch frequent benutzten Schimpfwörter. Eine allgemeine Abhandlung zu generellen Aspekten von Schimpfwörtern findet sich z. B. bei Havryliv (2003). S. auch Kiener (1983). 
b. Bezeichnungen aus dem (pseudo)religiösen und fiktiven Diskurs, die auf Wesen referieren, die das Böse per se personifizieren wie Teufel, Satan, AntiChrist, Unwesen, Unholde, Ungeheuer, Monster.

c. Schimpfwörter, die sich auf minderwertige Substanzen, krankhafte Auswüchse und Fäkalien beziehen wie Knochen, Mist, Dreck, Unrat, Geschwür, Pestbeule, Scheiße.

d. Benennungen für körperlich, intellektuell oder moralisch eingeschränkte bzw. als minderwertig erachtete Menschen wie Idiot, Missgeburt, Schwachkopf, Krüppel, Gehirnamputierter, Perversling, Heuchler, Lügner, Verbrecher, Mörder.

Diese Schimpfwörter kommen oft in Kombinationen vor: Adjektiv-Nomen-Phrasen wie mieses Judenschwein z. B. verbinden die pejorativen Komponenten der Dehumanisierung und der moralischen Schlechtigkeit, ein Kompositum wie in jüdische Teufelsbrut koppelt die dehumanisierende Semantik von Brut mit der religiös-metaphysischen Entwertung. Damit folgen die Beschimpfungen dem generellen Muster der Feindbildkonstruktion, die auf Dehumanisierung und Dämonisierung basiert (s. hierzu bereits Kap. 3). Wortzusammensetzungen, in denen die Lexeme Schwein, Dreck oder Mörder in erster oder zweiter Position stehen, sind in den Schreiben an den ZJD und die IBD besonders häufig eingesetzte Schimpfwörter: Judenschweine, Mörderbande, Kindermörder, Mörderbrut, Mördervolk, Krüppelvolk, Mörderpack, Drecksjuden, Schweinejuden, Dreckspack. Intensiviert wird die pejorative Komponente durch Adjektive wie stinkend, erbärmlich, widerlich, verlogen etc. Der Staat Israel wird bevorzugt mittels Komposita und metaphorischer Konstruktionen als Krebsgeschwür, Krüppelstaat, Kontergangebilde, Mörderwalze und Terroristennest bezeichnet.

Die häufigste Beschimpfungsvariante im Korpus umfasst elliptische Exklamative und Aufzählungen von einzelnen Pejorativa mit direkten Anredeformen:

$$
\begin{aligned}
& \text { „ihr Judenschweine“ [ZJD_08.09.2006_Mon_003] } \\
& \text { „Ihr Kinderfresser.“ [ZJD_Gaza2009_9/816_Hai_001] }
\end{aligned}
$$

„Ihr Terroristen und Kriegsverbrecher!!!!!!!!!!!!!!!“ [IBD_11.08.2011_ano_001]

$$
\begin{aligned}
& \text { „Ihr feigen Scheisshaufen!“ [ZJD_Gaza2009_677/816_Pan_001] } \\
& \text { „Ihr Kinderschlächter.“ [ZJD_Gaza2009_432/816_Hel_001] }
\end{aligned}
$$


(16) „Ihr Mörder und kriminelles Gangster-Regime [...] Ihr Kindermörder, Kinderfresser, Organklauer“ [IBD_04.01.2007_Ard_001]

(17) „Ihr dreckigen Juden und MOERDERBANDE.“ [IBD_05.08.2010_ano_001]

(18) „Ihr Voelkermoerder und Kriegstreiber.“ [IBD_06.05.2009_ano_001]

Jede Beschimpfung stellt eine Degradierung des Adressaten dar, die dessen Minderwertigkeit demonstrieren soll. Es sind Herabstufungen im kollektiven Wertesystem einer Gesellschaft.

Meistens werden die Adressaten geduzt: Diese Form der informellen und despektierlichen Anrede verstärkt den Ausdruck der Geringschätzung und fokussiert die Beleidigungsabsicht. Beispiele wie (19), in denen die Adressaten gesiezt werden, sind daher selten unter den Beschimpfungen:

$$
\text { „Sie Schufte, Sie Kriegs-Verbrecher!“ [IBD_03.04.2009_Kub_001] }
$$

$\mathrm{Zu}$ einem großen Teil werden die Adressaten, wird der Adressat auch gar nicht angesprochen. Dann bestehen die elliptischen Texte aus bloßen Beschimpfungen in Form von einzelnen Wörtern, Phrasen oder Wortaneinanderreihungen, die eine generische, also kollektiv referenzialisierende Lesart haben. Oft dient dabei der inflationäre Gebrauch von Interpunktionszeichen wie „!“ als Emphasesignal.

(20) „BlurünstigeMörder, kriminelle Faschisten, Landdiebe, Plünderer Menschliche-Organ Klauer“ [IBD_26.01.2007_Ard_001]

$$
\begin{aligned}
& \text { „Massenmörder!!!!!!!!!!!!!!!“ [ZJD_09.08.2006_ano_001] } \\
& \text { „Dreckige arrogante Juden!!!“ [IBD_06.05.2009_ano_002] } \\
& \text { „Nazi-Schergen!!!!!!!!!!!!!!!!!!!!!!!!“ [IBD_11.02.2011_ano_001] }
\end{aligned}
$$

Bei vielen Beschimpfungen werden Adjektive und Nomen benutzt, die seit Jahrhunderten tradiert werden und Floskelstatus haben, da sie Klischees bedienen: hinterhältig, feige, rachsüchtig, Parasiten, Teufel z. B. sind Lexeme, die schon habituell im Mittelalter zur Charakterisierung von Juden benutzt wurden (s. Kap. 4.1). Entsprechend finden sich viele Äußerungen, die solche negativen Eigenschaften 
als typisch für Juden prädizieren und damit aus Produzentensicht die alten Vorurteile explizit bestätigt sehen:

(24) „Offensichtlich sind das [= morden, Unfrieden stiften, Land rauben, d. Verf.] die Grundeigenschaften der Juden.“ [IBD_15.06.2009_ano_001]

„JUDEN SIND DIE SCHLIMMSTEN LÜGNER UND GRÖSSTEN VERBRECHER DIE ES AUF DER WELT GIBT. JUDEN SIND DIE URSACHE DES TERRORISMUS.“ [IBD_07.07.2006_ano_045]

(26) „Ihr Juden koennt es nicht lassen ... Euer Staat ist eine Missgeburt wie Ihr selber wisst. Schaut das ihr mit euren Nachbarn zurecht kommt. Aber ihr fühlt Euch ja als das auserlesene Volk.“ [IBD_12.06.2006_ano_001]

Während viele der Schreiben sehr kurz sind und nur aus einigen aneinandergereihten Schimpfwörtern bestehen, also primär dem emotionsgeleiteten Ausdruck von Hass und Wut dienen, sind Beschimpfungen in zahlreichen E-Mails und Briefen oft lediglich der Auftakt für weitere Verbal-Aggressionen, die aus Warnungen, Drohungen und Verwünschungen bestehen:

(27) „Schande ueber Euch..Ihr widerlichen Drecksschweine. Eines Tages seid ihr ENDLICH ausgerottet.. Ihr arroganten, egoistischen, grausamen, affektierten Menschen.. Wer hat Euch die Fehlinformation gegeben, das ihr besser seid als alle Anderen?“ [ZJD_Gaza2009_401/816_Jar_001]

(28) „Man sollte Euch Scheiss Juden auch die Kehle durchschneiden [...]“ [ZJD_04.05.2007_Mau_001]

(29) „Judenschweine! Schade dass ihr nicht alle bei Adolf verreckt seid. Jeden einzelnen müsste man sicherheitshalber vergiften, vergasen und vorsichtshalber mit 10 Kugeln erschießen. Ihr Wichser seit wie eine Seuche und deshalb gehärt ihr ausgerottet. Wir werden Euch Dreckschweinen Schaden zufügen an allen Stellen. Ihr seid ein Scheißvolk! Es ist im Fernsehen wunderschön anzusehen, ein Genuss, nach einem Bombenanschlag, eine Judensau, egal ob jung oder alt, wichtig ist nur, dass sie verreckt im Straßendreck liegt. Kaum jemand auf der Welt mag einen Juden, überall nur Ablehnung! Man kann nur hoffen, dass es noch viele von Euch Superlumpengesindel tödlich erwischt.“

[IBD_01.01.2006_ano_026] 
Auch das Lexem Jude wird von manchen Verfassern wie ein Schimpfwort bereits in der Anrede bzw. der Begrüßung gebraucht.

(30) „Jude Simon Stein, mit deiner Äußerung hast du [...]“ [IBD_23.07.2006_Hud_001]

$$
\text { „JUDE!!!!!!!!!!!!!!!!!!!!!!!!!!!“ [ZJD_23.10.2007_ano_001] }
$$$$
\text { „Shalon ihr Juden, [...]“ [IBD_22.04.2008_Pal_001] }
$$

Durch abwertende Attribute wie dreckig und verdammt sowie emotionsausdrückende Interjektionen wie Iiih, pfui wird der pejorative Effekt dabei noch verstärkt.

$$
\begin{aligned}
& \text { „Ihr dreckigen Juden!!!!!!!!!!!!!!!!!!!!!!!!!!!!!!“ } \\
& \text { [ZJD_Gaza2009_301/816_ano_001, anonymer Mehrfachschreiber] }
\end{aligned}
$$

$$
\text { „Oh, ihr verdammten Juden!“ [IBD_16.04.2009_Kub_001] }
$$

$$
\text { „wie könnt ihr nur so streitsüchtig sein. igitt“ [IBD_23.03.2008_ano_001] }
$$

$$
\text { „Pfui Teufel ihr JUDEN.“[IBD_27.05.2009_ano_001] }
$$

Das Lexem Zionismus (von seiner Denotation her 'die Bestrebung, Juden aus der Diaspora nach Israel zurückzubringen') wird in seinen verschiedenen grammatischen Varianten ebenfalls ausschließlich als Schimpfwort ${ }^{5}$ benutzt. Die pejorative Komponente ist bereits als usuell zu konstatieren: Verfasser aller politischen Richtungen benutzen das Wort mit Abwertungsabsicht in den verschiedensten Variationen wie Zionistenschweine, zionistischer Feind, zionistisches Dreckspack,

5 Diese kommunikative Praxis hat einen realen Hintergrund: Am 10. November 1975 erfolgte im Rahmen der UN die Verurteilung des Zionismus (vgl. Resolution der UN-Vollversammlung, UNGV Res. 3379), wobei behauptet wurde, „dass der Zionismus eine Form des Rassismus und der rassischen Diskriminierung ist“. Diese offizielle und institutionelle Entwertung des Zionismus wurde erst im Dezember 1991 auf Druck der USA mit Zustimmung Russlands wieder aufgehoben. Bis heute jedoch verweisen Antisemiten auf diese Resolution. Dass das Lexem Zionist bzw. Zionismus eine Bedeutungsveränderung erfahren hat, zeigt sich deutlich: Im gesamten Korpus findet sich kein einziges Textbeispiel mit einer neutralen oder positiven Lesart. Auch im Kommunikationsraum des Internets finden sich primär die pejorativ gemeinten Verwendungen (s. Schwarz-Friesel 2012a). 
zionistische Hure (gemeint ist Charlotte Knobloch), zionistischer GefangenenBefreiungsterror, zionistischer Staatsterror, Zionsbrut:

(37) „Zionistischer Größenwahn und Kriegsverbechen wie Sabra und Shatilla werden dann zu bewerten und zu richten sein.“

[IBD_14.07.2006_Bru_001]

„Menschenverachtung und Kaltschnäuzigkeit zionistischer Verbrecher erreichen gerade jetzt einen einsamen Höhepunkt, da diese Gangster ein zuvor jahrzehntelang terrorisiertes, beraubtes, geknechtetes, drangsaliertes und in den Hungertod getriebenes Volk im Gaza Streifen nach allen Regeln einer perversen und außer Rand und Band geratenen Kriegsmaschinerie vor den Augen der Weltöffentlichkeit einfach abschlachten.“ [ZJD_Gaza2009_399/816_Sne_001]

(39) „An die national-zionistische Mörderbande“ [ZJD_Gaza2009_686/816_Fra_001]

Besonders häufig wird das Wort allerdings von linken und linksextremen Schreibern artikuliert. Zionistisch wird hier quasi-synonym mit imperialistisch, kolonialistisch und faschistisch verwendet und taucht kollokativ besonders häufig in der Verbindung mit Wörtern aus dem semantischen Feld 'Terrorismus, Gewalt und Unterdrückung' auf. Die Kriegsmetaphorik ist dominant: Juden und Israelis werden unterschiedslos als aggressive, räuberische, Land enteignende Kapitalisten und Imperialisten charakterisiert, der Zentralrat der Juden wird entsprechend delegitimiert.

(40) „In meinen Augen sind sie mittlerweile nichts anderes als eine verboten gehörende Organisation die eigentlich keinerlei Daseinsberechtigung haben sollte da sie die Völkerwidrige Kriegstreiberei des Staates Israel in diesem Lande forcieren und jedwege Kritik mit Diskreditierung beantworten. PFUI!!!“‘[ZJD_02.09.2006_Man_001]

(41) „Kritik und Sanktionen für die zionistischen Kriegsverbrecher!“ [ZJD_Gaza2009_194/816_ano_001]

(42) „Dieser Krieg ist blanker Staatsterrorismus gegen ein fast wehrloses Volk.“[IBD_19.07.2006_Bec_001] 
Rechtsradikale Schreiber dagegen verwenden besonders oft Anal-Fäkal-Ausdrücke und greifen bevorzugt auf vulgäre Tier- und Unrat-Metaphorik zurück (s. hierzu Hortzitz 1999 und Pörksen 2005).

(43) „Widerwärtiges israelisches Pack [...] Ihr Rattenpack [...].“ [IBD_05.05.2007_Kol_001]

(44) „dreckeliger jaud zieht euch aus palästina zurück ihr stinkenden parasiten“ [ZJD_22.05.2007_Lan_001]

(45) „kranke, widerwärtige und ultrabrutale Wichser“ [IBD_10.06.2006_Bre_001]

(46) „BETREFF: stinkender, verpisster Judendreck.“ [IBD_11.01.2009_ano_001]

Es werden stets generische, d.h. alle Juden umfassende Referenzialisierungen wie in (47) vollzogen. Die dehumanisierende Semantik wird durch die Verwendung des unpersönlichen Pronomens es in der anaphorischen Wiederaufnahme intensiviert:

(47) „Der Jude ist kein Mensch, es ist eine Fäulniserscheinung“ [IBD_01.08.2006_Mar_001]

(48) „Unmenschen!! Tiere, Dreckschweine!“ [IBD_18.04.2011_ano_001]

Vielfach kommt es zu Lexem-Anleihen aus der NS-Ideologie wie Untermenschengesindel, minderwertige Untermenschen sowie Verweisen auf die „Endlösung“ im Holocaust (s. hierzu Kap. 6).

(49) „Sieg Heil UNTERMENSCHENGESINDEL !“ [IBD_19.01.2004_ano_001]

(50) „Jud Ade, Zyklon B tut nicht weh. Der Tag naht an dem das Welt Judemtum endlich ausgerottet ist. Wir vernichten Euch alle aber vorher müsst Ihr noch richtig leiden, erst dann werden wir Euch alle restlos vernichten.“[IBD_11.01.2009_ano_001]

Überblickt man allerdings die E-Mails und Briefe in der Zeitspanne von 2002 bis 2011, fällt auf, dass sich die Sprachgebrauchsmuster zunehmend vermischen. Redewendungen, die bislang nur von Neonazis und Rechtsradikalen benutzt 
wurden, finden zunehmend auch Verwendung in Schreiben von Verfassern aus dem linksorientierten Lager und aus der Mitte (wie die folgenden exemplarischen Beispiele von erklärten linken und (links)liberalen Textproduzenten, zum Teil mit akademischen Titeln, zeigen).

(51) „Subject: Widerwärtiges israelisches Pack. Na haben Eure FaschistenBrüder wieder ein paar Araber gemordet? [...] Nur Hass und Gewalt für Eurch. Und die Zeit, Ihr Rattenpack, arbeitet gegen Euch.“

[IBD_05.05.2007_Ler_001]

(52) „Zur Hölle mit dem erzkriminellen zionischtischen Pack in Palästina und seinen Spießgesellen in Europa und Nordamerika!“ [ZJD_Gaza2009_521/816_Mel_001]

(53) „Es ist für mich als Antifaschist bestürzend, wie der Sicherheitsstaat Israel und seine Eliten blindwütig immer wieder in unnötige Unmenschlichkeiten zurückfallen und $\mathrm{zu}$ staatsterroristischen Hampelmännern [...] degenerieren. Die Unmenschen Göbbels und Hitler lachen sich in der Hölle bestimmt ins Fäustchen.“ [IBD_01.06.2010_Göl_001]

Nicht immer gibt es explizite Anredeformen der zweiten Person mit $\mathrm{Du} / \mathrm{Ihr}$ oder Sie. Es gibt auch unpersönliche Referenzialisierungen als Beschimpfungen wie in (54). Hier wählt der Verfasser des Textes die Pronomina der dritten Person und stellt somit den Wahrheitsgehalt seiner Negativattribuierungen als allgemeingültigen Fakt hin:

$$
\begin{aligned}
& \text { „JUDEN SIE SIND DAS KREGSGESCHWÜR AUF UNSERER WELT! DER } \\
& \text { ABSOLUTE DRECK DER MENSCHHEIT. EINES TAGES KOMMT DER } \\
& \text { WIRKLICHE HOLOCAUST. STEIN à SO SIEHT EIN GANGSTER AUS. DEM } \\
& \text { GLAUBT SOWIESO KEINER WAS: MIT JEDEM AUFTRITT IN DEN MEDIEN } \\
& \text { KOMMT NUR HASS AUF. EIN PERVERSER LÜGNER.“ } \\
& \text { [IBD_01.08.2006_Mor_001, Postkarte] }
\end{aligned}
$$

Juden werden mittels des generischen Satzes kollektiv entwertet und der damalige Botschafter Shimon Stein als ein typisches Exemplar von JUDE negativ konzeptualisiert. So folgt diese „Argumentation“ dem deduktiven Muster „,vom Allgemeinen zum Spezifischen“. In (55) sind die Beschimpfungen zusätzlich mit der impliziten Drohung verbunden, den Kindern des Botschafters Gewalt antun zu wollen. 
„Hey du botschafterschwein, hast du vielleicht auch Kinder, die in berlin leben? Israelische Drecksau.“ [IBD_20.10.2003_ano_001]

Die meisten Beschimpfungen und Beleidigungen sind Kollektivattribuierungen mit generischer Lesart, d. h. nicht nur der Zentralrat bzw. die Botschaft als Adressaten sind gemeint, sondern alle Juden bzw. Israelis. Es gibt aber auch individuelle Beschimpfungen, bei denen ein spezifischer Adressat/die Adressaten persönlich angesprochen und anschließend durch beleidigende Aussagen negativ evaluiert wird/werden, wie in Beispiel (56), wo der damaligen Vorsitzenden des ZJD, Charlotte Knobloch, mit pseudo-religiösen Vokabeln Blasphemie und moralische Verkommenheit vorgeworfen wird.

„,aber Sie [...] sind eine ÜBERFÜHRTE GOTTESLÄSTERERIN, die den jüdischen Gott des Lebens permanent zu einem zionistisch-tribalistischen Gott der Zerstörung und des Todes umdeutet hat!“

[ZJD_Gaza2009_136/816_Kra_001]

Während rechtsradikale Schreiber bei persönlichen Angriffen fast ausschließlich Schimpfwörter benutzen wie in (57) und (58), formulieren Textproduzenten aus der Mitte Beleidigungen bevorzugt in Frageform wie in (59):

„verlogene Moralhure Knobloch [...] Die ranzige Moralschlampe Knobloch widert mich nur noch abgrundtief an! Und den Holocaust kann sie sich sonstwohin stecken!“ [ZJD_19.04.2007_Ber_001]

(58) „Das die juedische Zentralratshure es bei der fehlenden Berichterstattung ueber Gaza es auch nocht wagt zu bemerken das die Presse den zionistischen Staat nicht genug repraesentiert ist Ekel erregend zynisch.“ [ZJD_Gaza2009_267/816_ano_001]

(59) „Sehr verehrte Frau Knobloch [...] Die Frage ist: leisten Sie sich lhre Frechheiten mit Kalkül und aus der Stärke der Verschwörung heraus oder sind Sie schlichtweg senil?““[ZJD_31.08.2006_Hyp_001]

Der zuvor im Amt des Zentralratsvorsitzenden tätige Paul Spiegel wird verhöhnt ${ }^{6}$ und der Lächerlichkeit preisgegeben, indem seine kommunikative und berufliche Tätigkeit mittels pejorativer Lexik abgewertet wird:

6 Der Sprechakt des Verhöhnens wird hier als eine Sonderform des Beleidigens verstanden: Spezifisch an einer verhöhnenden Beleidigung ist, dass der Adressat mittels Ironie, Sarkasmus 

„Sehr geehrter Herr Spiegel, in Ihrer Eigenschaft als Vorsitzender des Zentralrats der Juden in Deutschland haben Sie sich schon mehrmals in Ihrer Wortwahl vergriffen. Ich kann ja verstehen, daß ein Tingel- tangel-Agent mit komplizierten Problematiken seine Schwierigkeiten hat. Aber könnten Sie sich in Zukunft nicht besser beraten lassen?“ [ZJD_08.04.2005_Hah_001]

Spezifisch für rechtsextreme Zuschriften sind neben der Verwendung pejorativer Bezeichnungen und Beschimpfungen in der Anrede (Pinkeljude, Drecksjuden) auch saloppe Begrüßungsfloskeln wie Hey, Hallo und die Verunglimpfung von Namen (Paulchen Spiegel oder Charlotte Knoblauch). Es ist eine typisch antisemitische Strategie, die Namen von Juden z. B. durch Verdrehungen und negative Deutungen zu verhöhnen und zur Stigmatisierung zu benutzen (vgl. hierzu ausführlich Bering 1989a und ${ }^{3} 1991$ ).

(61) „Betreff: lotte knoblauch. verpassen sie ihrer vorsitzenden endlich einen maulkorb. sie mischt sich zu oft in innerdeutsche angelegenheiten ein, wozu sie keinerlei berechtigung hat, denn das judentum ist und bleibt eine ungeliebte - aber mußgeduldete - religiöse minderheit. charlottchen sollte sich mehr um ihre mazze und sabat-verpflegung kümmern und ihre impertinente visage aus der deuschen presse raushalten.“ [ZJD_23.10.2007_Sch_001]

(62) „sehr geehrter herr wolffsohn, mit dieser aussage haben sie ihren namen alle ehre gemacht.“ $[13.05 .04-\mathrm{m}-\mathrm{U} 1]^{7}$

Insbesondere die Zentralratsvorsitzenden werden von Rechtsextremen häufig auch nur mit Vornamen angesprochen, womit eine herablassende oder abschätzige Haltung ihnen gegenüber kommuniziert wird:

$$
\begin{aligned}
& \text { „Damit kein falscher Eindruck entsteht, lieber Paul, [...]“ } \\
& \text { [ZJD_27.05.2002_Sor_001] }
\end{aligned}
$$

oder Wortspielen verspottet und ins Lächerliche gezogen wird.

7 Diese Texte stammen aus einer Sammlung von ca. 1.000 E-Mails und Briefen, die Michael Wolffsohn (ein Deutscher jüdischen Glaubens und Geschichtsprofessor an der BWU in München) im Frühjahr 2004 erhalten hatte, nachdem er in einem Interview mit Sandra Maischberger bei N-TV (in dem es um neue Methoden im Kampf gegen den internationalen Terrorismus ging) auch den Einsatz von Folter befürwortete (s. Schwarz-Friesel 2007: 353). 
(64) „Hallo Charlotte, da bin ich mal wieder, diesmal aus Berlin, meiner geliebten Reichshauptstadt, in der ich Deinem Vorgänger 'Paulchen' Spiegel immer so nahe war. Schade um ihn, denn zum Schluß hatte er seine Grenzen doch so klar erkannt !“ [ZJD_18.11.2006_Seg_001]

Spezifisch für rechtsextreme Schreiber sind auch Verweise auf die Rechtmäßigkeit der „Judenpolitik“ Hitlers („Hitler hatte Recht!“) und „sprechende Namen“, die auf revisionistische Bestrebungen hindeuten. Rechtsextreme verweisen zumeist auch auf ihre ausgeprägte Protesthaltung bzw. ihre politischen Überzeugungen oder Vorbilder wie in (66). In (65) wird dabei über das Lexem Ketzer die gesamte rechtsextreme Argumentationskette impliziert, der zufolge es in Deutschland ein Meinungstabu gebe, welches die sogenannten „Systemparteien $\operatorname{der} B R D^{\text {“ }}$ aufrecht erhalten, und dem man sich nur ketzerisch, also mit der Gefahr eines Märtyrertodes widersetzen kann. Dass die Bundesrepublik als illegitim und von einer angeblich immer noch präsenten alliierten Besatzungsmacht oktroyiert aufgefasst wird, ergibt sich daraus, dass der Schreiber in (67) die Rechtsnachfolge des Dritten Reiches für sich beansprucht.

$$
\text { „Mit germanischen Gruß Erik der Ketzer“ [ZJD_08.02.2007_Wol_001] }
$$$$
\text { „freundlichst, ein anhänger Henry Fords“ [ZJD_22.03.2007_ano_001] }
$$

„Für das Deutsche Reich in Geschäftsführung ohne Auftrag handelnd“ [IBD_08.11.2006_Pat_001]

Beschimpfungen und Beleidigungen sind nicht immer strikt voneinander abzugrenzen. Beides sind Formen verbaler Gewalt, die das Ziel haben, die Würde und die Integrität des Adressaten anzugreifen bzw. zu beschmutzen. Beschimpfungen sind im Grunde genommen die primitivste Form der Beleidigung, es sind affektive Aggressivitäts- und Hassbekundungen ohne argumentative Inhalte. Die Herabwürdigung des kommunikativen Gegenübers erfolgt ausschließlich über den Ausdruck stark pejorativer Lexeme. Beleidigungen können dagegen auf einer elaborierten Argumentation basieren und müssen nicht notwendigerweise mittels Schimpfwörter erfolgen. Auch eine ironische Äußerung wie in (68), Sarkasmus wie in (69), eine rhetorische Frage wie in (70) und (71) oder eine diffamierende Feststellung wie (72), teils mittels unangemessener Analogien wie in (73) und (74), können beleidigend sein. 
(68) „Die Armee des Mörder-, Folter- und Landraubstaates Israel hat wieder einen gloriosen Sieg über Steine werfende palästinensische Kinder und Jugendliche errungen.“ [IBD_28.03.2004_Wul_001]

(69) „Glückwünsche an die israelischen Brüder und Schwestern“ [ZJD_05.01.2009_Nie_001]

(70) „ISRAEL - EIN VOLK VON MÖRDERN???““ [IBD_19.03.2009_Müh_001]

(71) „Seid ihr noch normal?““[ZJD_09.01.2009_Cap_001]

(72) „Israelische Soldaten sind Mörder!“ [ZJD_Gaza2009_87/816_May_001]

(73) „Was habt ihr gut von den Nazis gelernt !!!“ [IBD_20.03.2008_Voc_001]

(74) „Ihr ermordet Palaestinenser wie es euch gefaellt, wie es frueher die KZ-Aufseher mit euch gemacht haben. Nur die wurden bestraft!“ [IBD_20.04.2009_ano_001]

Beleidigungen können auf komplexen stereotypen Inhalten und pseudo-rationalen Argumentationsketten beruhen, die alle der Ab- und Entwertung des Adressaten dienen und stets NS-Vergleiche involvieren:

(75) „Sie werben für Solidarität für Israel. Ich kann nur sagen: Nieder mit den Juden! Sie haben aus Gaza ein Konzentrationslager gemacht. Seit Jahrzehnten sperren Sie die Einheimischen ein, haben ein Apartheid-System geschaffen - da ist es kein Wunder, wenn Widerstand bzw. Terrorismus entsteht. Wie sagte doch MP Andreotti so treffend: 'Wir wären alle Terroristen, wenn wir in einem Konzentrationslager wie Gaza aufwachsen und ohne irgendeine Perspetive leben müßten.' Stimmt. Geben Sie die Besatzung auf! Dann wird sich vielleicht etwas ändern. Was kann ich tun? Ich boykottiere strikt alles Jüdische - und spucke ich vor Ihnen aus!“ [ZJD_Gaza2009_468/816_Kub_001]

(76) „Die Westbank wird genauso in Schutt und Asche gelegt wie Gaza. Die Menschen werden dort genau so abgeschlachtet wie in Gaza. Von Frieden keine Spur, weil SIrael gnadenlos alles niedermetzelt was palästinensisch. Vor allem die Bestien von Siedlern, die der größte israelische Abschaum sind, dürfen tun und lassen was sie wollen. Ermorden von Palästinensern, Gewaltakte gegen Palästinenser ausführen, Kinder der 
Palästinenser misshandeln, Ernten vernichten und Eigentum der Palästinenser anzünden, Land rauben, Und vieles mehr. Aber die israelische Regierung ist nicht anders. Sie lässt die Palästinenser verhungern, verdursten, verweigert medizinische Hilfe, raubt permanent das Land der Palästinenser, vernichtet Eigentum der Palästinenser, schlachtet die Palästinenser ab (egal ob alt oder jung, egal ob Mann , Frau oder Kind) sie lässt Palästinenser entführen und foltern und in Konzentrationslager sperren, die den KZ der Nazis in nichts nachstehen.“

[IBD_18.10.2007_Dro_001]

Beleidigend sind aber auch Äußerungen, die den/die Adressaten in unangemessener Weise belehren und maßregeln sollen und somit eine asymmetrische Kommunikationssituation konstruieren derart, dass die Adressaten als intellektuell, emotional und moralisch minderwertigere Personen disqualifiziert werden wie in „Haben Sie keinen Funken Mitleid in sich?“, „Humanisten verachten Sie zu Recht!“, „Kommen Sie endlich zur Vernunft!“ Diese subtileren Formen der Beleidigung werden vor allem von Verfassern aus der Mitte der Gesellschaft und von Akademikern, die sich selbst als linksliberal, nicht antisemitisch und besorgt bezeichnen, ${ }^{8}$ verwendet (s. hierzu 10.2 in diesem Kap.).

Drohungen beinhalten stets eine Handlungsankündigung des Produzenten: Die angekündigten Handlungen sind dabei für den Rezipienten immer negativ. Spezifische Drohungen kündigen ein partikulares Verhalten, unspezifische Drohungen eine nicht näher erläuterte zukünftige Aktion oder Bestrafung an:

(77) „Ich jedenfalls werde meinen Mund bestimmt nicht halten und die Verbrechen der Israelis, also der Juden, überall öffentlich machen.“ [IBD_04.07.2007_Dro_001]

(78) „aber Ihr werdet die Rechnung noch kriegen.“ [ZJD_30.05.2002_Kre_001]

(79) „IHR WERDET EURE POLITIK NOCH BEREUEN , da kann auch Staatsterrorist Bush und Vasall(in) Merkel nicht mehr helfen !“

[IBD_13.07.2006_Fro_001]

8 Hiervon abzugrenzen sind Schreiben, deren Verfasser tatsächlich besorgt sind und in genuiner Ratlosigkeit beim Zentralrat und der Botschaft entweder um eine Auskunft bitten oder Fragen stellen. Diese Schreiber verzichten auf maßregelnde Moralappelle, die implizieren, dass Juden und Israelis kognitiv und affektiv minderbemittelt seien. Vgl. hierzu die Beispiele in Kap. 7.1.2. 
(80) „Sie werden für diese Morde, für jedes einzelne Kleinkind, dass sie ermordet haben, für das, was sie Menschen angetan haben, werden sie büßen...sei es hier auf Erden sei es im Reich der unendlichen Gerechtigkeit... Aber wir werden nicht warten bis zum jüngsten Tag.“

[IBD_12.02.2009_ano_001]

Solche Kommissiva, also Sprechakte, mit denen sich der Produzent auf eine zukünftige Handlung festlegt, setzen typischerweise voraus, dass der Verfasser der Äußerung in der Lage ist, seine angekündigte Handlung auch auszuführen. Dies ist jedoch bei den angedrohten Maßnahmen der Schreiber in Bezug auf den ZJD und die IBD nicht immer als gegeben anzusehen. Daher sind reale und irreale Drohungen zu differenzieren. Explizit taucht der Sprechakttyp der realen (An)Drohung vor allem in Form von Boykottdrohungen, Protest- und Widerstandsankündigungen sowie Aufwiegelungsaktionen auf.

(81) „Ab sofort werde ich keinerlei Waren mehr kaufen, die aus Ihrem Land kommen! Und ich werde alle meine Bekannten und Freunde auffordern es genauso $\mathrm{zu}$ machen [...]““ [IBD_13.07.2006_Fis_003]

(82) „Zumindest im Kleinen werde ich alles tun, um Ihren Staat nicht zu unterstützen. Ich werde weder Produkte kaufen, die für mich erkennbar aus Israel kommen, noch werde ich jemals einen Gedanken daran verschwenden meinen Urlaub im 'Heiligen' Land zu verbringen. Ich hoffe nur, dass ich damit Vorbild für viele Andere sein kann.“

[IBD_05.06.2010_Hüb_001]

(83) „Auch ich werde mich ab sofort an Anti-Israel-Demonstrationen beteiligen.“[IBD_16.01.2009_Kel_001]

(84) „So lange es nicht eine wirklich zum Frieden bereite Regierung gibt, werde ich nie mehr nach Israel reisen und alles tun, diese Politik, die den wichtigen und notwendigen Freidensprozess blockiert, anklagen. Als Journalist gibt es diese Möglichkeit häufig.“ [IBD_02.06.2010_Fra_001]

(85) „Ich werde alles tun, um meine Mitmenschen gegen Israel aufzuhetzen.“ [ZJD_Gaza2009_799/816_ano_001]

Diese Boykott- und Protestandrohungen liegen im Handlungsspektrum des Möglichen. Entsprechend ist es mit Ankündigungen, die ein aktives Verhalten in Aussicht stellen. Dabei unterscheiden die meisten der Schreiber nicht zwischen 
israelischen und jüdischen Belangen: Die Juden in Deutschland werden kollektiv verantwortlich gemacht für israelische Militäroperationen etc. (wie die folgenden E-Mails an den Zentralrat belegen):

(86) „Wie bereits angemerkt bin ich kein Freund von Gewalt, aber den friedlichen Protest gegen Ihr Vorgehen werde ich mir nicht nehmen lassen.“ [ZJD_Gaza2009_723/816_Kuh_001]

(87) „Ich boykottiere seit Jahren alles Jüdische - und so wird das auch bleiben. Ich wünsche Ihnen alles erdenklich Schlechte!“

[ZJD_05.12.2008_ano_001]

Irreale Drohungen dagegen beziehen sich auf Handlungsszenarien, die entweder vom Verfasser nicht zu realisieren sind, s. (88), oder Bezug auf fiktive Domänen nehmen, s. (89):

(88) „Und ich gehöre zu der Generation,die das Glück haben,Israel verrecken zu sehen.Und wenn Israel stirbt,wenn eure Städte zerfallen und eure Bevölkerung verreckt-werde ich auf die Knie sinken,demütig die Hände falten und werde Gott danken-für SEINE Gerechtigkeit. Fahrt zur Höllewo ihr hingehört.Denn ihr seid das Böse dieser Welt.Es wird sehr schön euch verrecken zu sehen!Ich danke Gott dafür-das erleben zu dürfen.“ [IBD_09.05.2010_ano_012]

(89) „wo es irgend möglich ist werden sie bekämpft werden bis sie in einer 'hölle' untergehen.“[IBD_26.07.2006_Mor_001]

Die Drohungen sind oft an Aufforderungen mit Bedingungen der Art „Wenn nicht $\mathrm{x}$, dann y“ gekoppelt: Dabei wird das zukünftige Eintreten eines Umstandes, der vom Produzenten antizipiert wird, vom Verhalten des Adressaten abhängig gemacht, s. (90) und (91):

(90) „Ich bin kein Prophet und kenne die Nahost-Probleme nicht. Es steht mir auch nicht zu, mich in die 'inneren' Probleme anderer Staaten einzumischen. Eines glaube ich aber zu wissen: Freiheit ist unteilbar. Wenn Israel dies nicht anerkennen will, werden die Folgen nicht ausbleiben.“ [IBD_29.03.2004_Sta_001] 
„aber wenn nicht sofort ein Waffenstillstand von Seiten Israels ausgesprochen wird, werden wir nicht nur gegen die NPD sonder auch für den Frieden und damit [...] Israel demonstrieren!“ [ZJD_30.07.2006_Wel_001]

Auffällig ist bei solchen an Bedingungen geknüpften Drohungen, dass dem Zentralrat der Juden in Deutschland immer die moralische Integrität abgesprochen wird, wie in (92).

„Entweder Sie verhalten sich respektabel und werden dann auch entsprechend behandelt, oder man wird nie Verständnis für Ihre Religion und Ihr Volk aufbringen.“ [ZJD_Gaza2009_723/816_Kuh_001]

Die missionarisch auftretenden Schreiber artikulieren dabei wie strenge Schulmeister Anstands- und Benimmregeln (s. 10.2 in diesem Kap.). Diesem kommunikativen Verhalten liegt die Einstellung zugrunde, Juden seien selbst nicht in der Lage, ethische Prinzipien zu erkennen und $\mathrm{zu}$ realisieren. Die direkt auf Israel bezogenen Drohungen lassen meist eine Feindseligkeit erkennen, die auf der Konzeptualisierung beruht: ISRAEL IST UNABHÄNGIG VON SEINEN TATEN OHNEHIN ZU VERDAMMEN.

(93) „Wenn Israel weiter so agiert, hat Israel keine Überlebenschance. Und das ist dann auch gut so.“ [IBD_16.01.2009_ano_006]

(94) „Ich fürchte, wenn Israel sich nicht bewegt, wird es im mangelnden Realismus 'als auserwähltes Volk' seinen Untergang bewirken.“ [IBD_11.05.2010_Sta_001]

(95) „die betonköpfe in israel,werden den judenstaat an den abgrund seiner exestenz bringen,wenn nicht die h i t l e r r e g i e r u n g in israel gewaltsam,beseit4t wird!“ [ZJD_Gaza2009_12/816_ano_001]

Indem der Produzent die Realisierung des Angedrohten von bestimmten Bedingungen abhängig macht, will er Druck ausüben und den Rezipienten zwingen, etwas zu tun oder zu unterlassen. Schreiber, die den ZJD und die IBD verbal auf diese Weise bedrohen, sind von der Rechtmäßigkeit ihrer Forderungen überzeugt und wollen dem kommunikativen Gegenüber verbal ihren Willen aufzwingen. Diese Drohungen richten sich nach der Devise „und bist du nicht willig, so brauch ich Gewalt ... “ und offenbaren auf der Produzentenseite die tradierte Konzeptualisierung JUDEN SIND UNEINSICHTIG UND VERSTOCKT und WIR HABEN DIE PFLICHT UND VERANTWORTUNG, IHNEN DIE AUGEN ZU ÖFFNEN, SIE ZU LÄUTERN, 
AUCH MIT GEWALT. Expressis verbis wird diese Gedankenkette z. B. in (96) artikuliert. Diese Drohung beinhaltet nicht nur eine zukünftige Gewaltankündigung als moralische Verpflichtung, sondern auch einen globalen Lösungsvorschlag (der in Analogie zur nationalsozialistischen „Endlösung“ gemacht wird):

(96) „Um die Juden wieder zu Verstand zu bringen ist es die Pflicht eines jeden zivilisierten Menschen diese erbaermlichen Auserwaehlten zu schlagen, $\mathrm{zu}$ hauen $\mathrm{zu}$ stechen oder einfach von ihrem Wahn $\mathrm{zu}$ erloesen. [...] Am Ende wird es wieder eine diemal hoffentlich gruendliche 'Kristallnacht' geben.“[ZJD_Gaza2009_676/816_Pan_001]

Viele Drohungen stehen nicht allein, sondern sind an Beschimpfungen und Gefühlsbekundungen gekoppelt:

(97) „Wie lange wollt ihr scheiss Juden eigentlich noch unschuldige Zivilisten ungestraft töten. Für jeden toten Libanesen müssten 1000 von Eurer Sorte verrotten. Ich kann nur hoffen das für Euch irgendwann die Kammern wieder geöffnet werden. Ihr kotzt mich einfach nur an. Jedenfalls, für jeden toten von Euch lass ich mir ein Glas extra schmecken. Und seid Euch sicher,dies ist keine Einzelmeinung.“ [ZJD_07.08.2006_ano_001]

Der (das Schreiben des Verfassers legitimierende) Hinweis auf die Repräsentativität der Meinung verstärkt das Drohungspotenzial.

Bei rechtsextremen Verfassern bestehen die Drohungen in Ankündigungen exzessiver körperlicher Gewalt. Obszönitäten und derb-vulgäre Lexik sind dominant. Dieser Vulgärantisemitismus ist typisch für die Artikulation des Judenhasses durch Neonazis und Rechtsextreme (auch im Internet, wie (101), ein Text von der Informationsplattform StudiVZ, zeigt).

(98) „Euch Schweinen werden wir die Hoden abreissen und die Fotzen verbrennen!“[ZJD_Gaza2009_677/816_ano_001]

(99) „Man sollte Euch Scheiss Juden auch die Kehle durchschneiden, aber da käme an Stelle von Blut nur Stinkende Scheisse raus.“

[ZJD_04.05.2007_Mau_001]

(100) „Fuck Israhell““ [ZJD_09.01.2009_Cap_001]

(101) „Ich werde euch im KZ Auschwitz feierlich vergasen! Eure Frauen werde ich zu Tode vergewaltigen. Das wird ein Genuss! Ihr elendiges Pack 
bekommt genau das, was ihr verdient. Das deutsche Volk wird aus dieser ethnischen Säuberung groß und gereinigt hervorgehen! Ihr jüdisches Pack! Judentum ist Verbrechertum. Juden sind zum töten da“ [studiVZ am 02.08.2008 um 18:33 Uhr; mittlerweile im Internet gelöscht]

Diese irrealen Drohungen spiegeln extreme Gewalt- und Mordphantasien der Verfasser wider und sind als Ausdruck ihres hasserfüllten Wunschdenkens zu betrachten. Bei Verfassern mit rechtsradikaler Gesinnung werden die Drohungen (oft mit dem Hitlergruß) stets zusammen mit Beschimpfungen kodiert, die erkennbar Lexik aus der NS-Zeit enthalten:

(102) „Sieg Heil UNTERMENSCHENSGESINDEL! So wie es aussieht haben unsere Großvaeter doch noch nicht gruendlich genug gearbeitet! Wird wieder einemla Zeit das richtige Arier den Gashahn aufdrehen! Hey Paul Spiegel kannst du uns hoeren Drecksjude???Der Tag der Abrechnung wird kommen-Sieg Heil und dann bekommt ihr euren Holocaust den es bis JETZT noch nie gegeben hat....hahah...aber was noch nicht ist kann a noch werden! HEIL HITLER!“ [ZJD_19.01.2004_ano_001]

(103) „Ich gestatte mir noch einmal darauf aufmerksam zu machen, daß ich mit sofortiger Wirkung jeden Juden der mir über den Weg läuft absteche wie ein Schwein. Heil Hitler [...]“ [IBD_26.04.2009_Kru_001]

Die Drohungen von gebildeten Schreibern, wie die eines promovierten Akademikers in (104), unterscheiden sich allerdings oft nur marginal vom Vulgärantisemitismus der Rechtsextremisten:

(104) „Wir bringen Euch nach Den Haag, morgen, in einem Jahr, vielleicht auch erst sehr viel später! Ihr, die Ihr von Deutschland aus Benzin in den Tank der IDF-Bestien füllt, die Bevölkerung manipuliert und Euch so biedermännisch gebt, Ihr seid Brandstifter, Mordkomplizen. Gaza ist Warschau, die IDF ist die Wehrmacht und die SS und auch Livni, Barak und Olmert sind Reinkarnationen! Ihr kommt nicht ungestraft davon, Den Haag wartet!“ [ZJD_Gaza2009_154/816_Haf_001]

Aggressive Ankündigungen, die auf die Zukunft bezogen sind, lassen sich als prophezeiende Drohungen charakterisieren:

(105) „Ich mache Ihnen nun eine Prophezeiung, Herr Botschafter. Der Staat Israel wird von nun an beginnen auseinanderzufallen, er wird zerbre- 
chen. Ihr werdet anfangen, Euch gegenseitig zu hassen für das, was Ihr verbrochen habt.“ [IBD_13.07.2006_Hel_001]

(106) „Es wird eine Zeit kommen, da werden die Israelis für ihre Kriegsverbrechen zur Rechenschaft gezogen.“[IBD_16.01.2009_Kel_001]

(107) „Für da alles werdet ihr noch zur Rechenschaft gezogen und dafür werdet ihr sterben.“[IBD_03.03.2008_Mie_001]

(108) „Sie werden vor der Gerechtigkeit der Menschheit, vor der Justiz von Rechtsstaaten, die ihre Greueltaten nicht billigen, vor Gericht gestellt und verurteilt werden.“[IBD_12.02.2009_ano_001]

(109) „Aber vergisst eins nicht: 'Israel wird von der Landkarte gelöscht.'“ [IBD_28.05.2010_Özd_001]

Es werden Aussagen über zukünftige negative Ereignisse gemacht, die den Adressaten betreffen. Vorausgesagt werden juristische und metaphysische Bestrafungen, die als gewiss angesehen werden. Die Verfasser solcher Drohprophezeiungen haben zugleich das Bedürfnis, dem Adressaten eine unheilvolle Zukunft vorherzusagen. Die Grenze zwischen solchen Drohbekundungen und den Sprechakten der Verwünschung ist daher fließend. ${ }^{9}$ Vgl. zu Phantasie-Aggressionen allgemein auch Kiener (1983: 220).

(110) „Betreff: Ihr gottverdammten Hurensöhne. Wagt es nie mehr gegneüber uns uns Deutsche in der 2. Generation des Naziregimes euren moralischen Zeigenfinger wegen Verbrechen unserer Grossväter zu erheben, ihr Kinderschlächter. Ich hoffe inständig, das Iran wirklich über die Atombombe verfügt und eurem verlogenem Treiben endgültig ein Ende bereitet. Wennn dieses Schreiben zu rechtlichen Konsequenzen führen sollte, habe ich endgültig einen Grund, aktiv gegenüber euren Lügen und Morden an einer eingesperrten Zivilbevölvkerung vorzugehen. Ich wün-

\footnotetext{
9 Bei Drohungen (auch irrealer Art) verpflichtet sich der Sprachproduzent dafür zu sorgen, dass sich die angekündigten Strafmaßnahmen etc. erfüllen; er stellt deren zukünftige Realisierung als sicher dar; bei Verwünschungen setzt der Kommunizierende dagegen darauf, dass „höhere Mächte“ das Unheil über den Rezipienten bringen (sollen). Drohungen sind Ankündigungen, Verwünschungen (wie die Bestandteile der Wortbedeutung es angeben) sind Wunschvorstellungen der Sprachproduzenten.
} 
sche euch die Pest und alles Schlechte an den Hals.“

[ZJD_Gaza2009_432/816_Hel_001]

Verwünschungen (hier synonym mit Verfluchungen ${ }^{10}$ ) sind Äußerungen, in denen der Wunsch zum Ausdruck gebracht wird, dass dem Adressaten ein Unglück widerfährt. Es handelt sich um einen bestimmten Typ der Kommissiva. Obwohl sich die Verfasser nicht selbst zu der Handlung verpflichten, dem Adressaten ein Leid anzutun, haben die Verwünschungen eine ähnliche kommunikative Funktion wie Drohungen: Ziel dieser Sprachhandlung ist, neben dem Ausdruck der Entwertung, beim Rezipienten Furcht und Sorge zu aktivieren. Verwünschen bezieht sich auf den Rezipienten, sein Umfeld etc., denen Unheil gewünscht wird und denen etwas Schlimmes passieren soll.

(112) „So spricht der Herr Israel du hast gemordet seit 3000 Jahren und fremdes Eigentum geraubt. Hunde sollen dein Blut lecken. Gott will Unheil über dich bringen und Dich vertilgen ? deine Nachkommen will ausrotten was männlich ist bis auf den letzten Mann so Gott will“ [IBD_15.07.2006_ano_Postkarte]

(113) Saubande, hol Euch der Teufel ihr elenden Kriegstreiber.“ [IBD_12.07.2006_ano_Postkarte]

(114) „Mein Wunsch ist: Das endlich die arabischen Völker sich gegen euch verbünden und euch in die Hölle schicken woher ihr hergekommen seid. Das 'Rote Meer' soll seinen Namen verdienen.“ [ZJD_Gaza2009_792/816_ano_001]

(115) „Sollen eure Felder verdorren.“ [IBD_30.05.2007_ano_001]

(116) „Diese Verbrechen übersteigen jeden Anderen in Menschlicher Geschichte! Herr Stein Ihr zynische Haltung, Ihre Freude an Tod, Hungern, Morden, Foltern, Gefangenhalten und Plündern des palästinensischem Volk wird hoffentlich Ihnen jegliche Ruhe, Gesundheit und Glück nehmen. Ich wünsche Ihnen ein langes elendes unglückliches

10 Verwünschungen/Verfluchungen beziehen sich auf den/die Adressaten. Fluchen kann wie Schimpfen rein situations- und objektbedingt sein und bezieht sich auf ärgerliche Situationen (Verflucht, zum Teufel, das ist doch wie verhext, verdammt). 
Leben. Alles, was Sie dem Palästinensischen Kindern angetan haben, möge 1000 Fach Ihnen geschehen.“ [IBD_24.01.2007_Ard_001]

(117) „Weg mit Euch, [...] Ihr lügenhaften Engel, weg mit Euch. Die Erde soll sich auftun und Euch verschlucken, daß keine Spur von Euch bleibt und all Eure Mauern, Panzer, Bomber, Bulldozer sollen verschwinden. Fort mit Euch! Es lebe Palästina in Israel und Isra3el in Palästina“ [IBD_18.07.2006_Bil_001]

(118) „Ich wünsche dem ZR der Juden, dem auserwählten Tätervolk von USrael, den jüdischen Terroristen,Kriegsverbrechern und deren Unterstützern für das neue Jahr alle Seuchen,Sprengstoffanschläge und Schlechtigkeiten dieser Welt an den Hals.Möget Ihr leiden ,schliesslich seid ihr ein selbsternanntes auserwähltes Volk.“[ZJD_Gaza2009_754/816_ano_001]

Auch Akademiker aus der Mitte der Gesellschaft greifen manchmal auf Verwünschungen zurück und leben damit genau die atavistische Rachsucht aus, die sie sonst kollektiv den Juden unterstellen, wie die E-Mails eines promovierten Mediziners, s. (119), und die eines Rechtsanwalts, s. (120), zeigen:

„[...] soll auch Israel keine Ruhe finden und in Angst vor den nächsten Attentaten leben.“[ZJD_24.06.2002_Mau_001]

„Gott wird Sie dafür strafen!“ [IBD_05.02.2009_Kel_001]

Bei Verwünschungen handelt es sich um verbale Manifestationen von Hass und Aggression, die zugleich das Ausmaß ohnmächtiger Wut widerspiegeln. Auf Verwünschungen greift man zurück, wenn sich in der realen Welt keine Möglichkeit bietet, dem Hassobjekt tatsächlich Schaden zuzufügen.

(121) „Ich wünsche Ihnen alles erdenklich Schlechte!“

[IBD_20.03.2009_ano_001]

(122) „Ich wünsche euch ein langes Leben voller Schmerz, Qual, Schmach und Erniedrigung, jeden Atemzug soll euch Folter ohne Gleichen sein.“ [IBD_04.01.2007_Ard_001]

(123) „Hoffentlich krepiert ihr alle jämmerlich!“

[ZJD_Gaza2009_301/816_ano_001] 
(124) „Ich hoffe inständig, das Iran wirklich über die Atombombe verfügt und eurem verlogenem Treiben endgültig ein Ende bereitet.“

[ZJD_Gaza2009_432/816_Hes_001]

(125) „Es ist zu hoffen, daß Israel genügend Tote $\mathrm{zu}$ beklagen hat, wie Europa im 30-jährigen Krieg, um (im wahersten Sinne des Wortes) zur Vernunft zu kommen.“[ZJD_Gaza2009_552/816_Mad_001]

In (121) bis (125) artikulieren die Verfasser unter Verwendung der expressiven Verben wünschen bzw. hoffen sowie des Satzadverbs hoffentlich ihre positive Einstellung zu den grauenvollen Dingen, die sie dem Adressaten wünschen. Solche Satzkonstruktionen weisen eine Doppelproposition auf: Es handelt sich um Sätze, bei denen die Proposition von einem emotiven Ausdruck, der die emotionale Einstellung des Produzenten und damit zugleich eine Bewertung ausdrückt, determiniert wird. Die lexikalische Bedeutung eines solchen Satzes beinhaltet damit einerseits diese spezifische Einstellung, andererseits die Sachverhaltsproposition, auf die sich der Einstellungsausdruck bezieht. Semantisch haben wir somit eine Doppelproposition: Eine Proposition in einer Einstellungsproposition, EP (P) (s. hierzu Schwarz-Friesel 2007: 174).

In Verwünschungen nehmen die Schreiber häufig Bezug auf höhere Mächte wie den Himmel oder die Hölle: Die Hölle soll euch verschlucken, Gott wird euch strafen. Häufig werden hierbei idiomatische Wendungen benutzt wie die Pest an den Hals wünschen und der Teufel soll euch holen. Floskelhafte Sprichwörter werden ebenfalls in verschiedenen Abwandlungen hierfür herangezogen: Wer Gewalt sät, wird Gewalt ernten, Wer so viel Hass sät, wird selber Hass erhalten, Wer die Saat des Hasses pflanzt, soll selbst Hass ernten.

„'Wer Haß säht, wird Haß ernten'.“ [IBD_01.08.2006_Ren_001]

„Hass sät Hass, das heutige Israel ist im Blutschlamm des Alten Testaments stecken geblieben.“ [IBD_01.06.2010_Göl_001]

Verwünschungen haben Ventilfunktion für die exzessiven Gefühle der Schreiber (s. hierzu Kap.9). Es sind emotions- und affektgesteuerte sowie atavistische Kommunikationsformen; sie sind atavistisch in dem Sinne, dass sie auf dem Glauben an die reale Kraft von Sprachmagie basieren. Sie entsprechen exakt dem judeophoben Ressentiment: Sie drücken einerseits den tiefen Hass und Groll gegenüber Juden aus, sind aber auch Indikatoren für die als ohnmächtig empfundenen Wutgefühle der Verfasser: Wenn die Produzenten im realen Lebensbereich nichts gegen die „verdorbenen Judenschweine“ auszurichten vermögen, so sollen 
wenigstens die Flüche wie ein Voodoo-Zauber ihre Wirkung entfalten. Dem unbedingten Wunsch, den verhassten Juden Schaden zufügen zu wollen, aber dies im realen Leben individuell nicht zu können, verleiht zumindest die Sprache mit ihrem semantischen Zerstörungspotenzial Ausdruck.

\subsection{Judenfeindschaft als Missionarsdrang: Moralappelle und Ratschläge}

Artikuliert sich der extremistische Antisemitismus mittels Hassausdruck und Gewaltphantasien als ostentative Aggressivität, kodieren Verfasser der Mitte ihre judeophobe Einstellung eher auf verbalen Umwegen, d. h. mittels Re-Kodierungen von Sprachhandlungen als „,besorgte Kritik“, „Entrüstung“ und „moralische Verpflichtung“ (s. hierzu die Strategien der Legitimierung und Abschwächung, Kap.11.2 und 11.3). Stellen Extremisten Juden prinzipiell als minderwertige, verkommene Wesen eines verbrecherischen Volkes dar, so verweisen Textproduzenten aus der Mitte dagegen auf die insinuierten intellektuellen, affektiven und moralischen Schwächen der jüdischen Mitbürger sowie Israelis und treten kommunikativ wie Lehrer, Richter und/oder Ratgeber auf. Bei Verfassern aus der Mitte der Gesellschaft werden daher vor allem erzieherische Direktiva im Imperativ artikuliert, mit denen die Adressaten aufgefordert werden, sich zu schämen und/oder endlich zu bessern:

$$
\begin{aligned}
& \text { „[...] schämen sie sich!“ [IBD_05.02.2009_Mar_001] } \\
& \text { „[...] und schämen Sie sich!““ [IBD_03.04.2009_Kub_001] } \\
& \text { „[...] schämt EUCH!!!“ [ZJD_Gaza2009_306/816_ano_001] }
\end{aligned}
$$

„Abschliessend möchte ich noch einmal an das für mich wichtigste Gebot Moses erinnern : Du sollst nicht töten. Haltet euch dran, verdammt.“ [ZJD_Gaza2009_14/816_ano_001]

Die Textproduzenten sehen sich selbst offensichtlich in einer dem Rezipienten hierarchisch übergeordneten Position, die ihnen eine derartige Aufforderung erlaubt. Die Aufforderung Schämt euch! ist vor allem im situativen Kontext der Kindererziehung gebräuchlich und impliziert, dass es auf Rezipientenseite einen Grund gibt, sich zu schämen, nämlich schlechtes Benehmen. Es handelt sich um Maßregelungen, denen eine asymmetrische Kommunikationskonstellation zugrundeliegt, bei der der Produzent aus seinem Überlegenheitsgefühl heraus 
den Rezipienten ermahnt. Es kommt in zahlreichen Schreiben zu dem Versuch der Verfasser, dem ZJD vorzuschreiben, was er tun oder unterlassen soll. Insbesondere wird immer wieder eine kritisch distanzierte Einstellung zu Israel angemahnt.

(132) „Warum aber meldet sich der Zentralrat der Juden in Deutschland nicht ebenfalls kritisch zu Wort und verurteilt das offensichtliche Unrecht, [...] Warum findet der Vizepräsident Ihrer Organisation gegen den aggressiven Krieg Israels gegen Palästina, der keine einfache Verteidigung gegen Terrorismus mehr ist, sondern selbst terrorisiert?“

[ZJD_09.04.2002_Sto_001]

(133) „Warum distanzieren Sie sich nicht von dem mörderischen Krieg des Staates Israel gegen die Zivilbevölkerung Palästinas?“

[ZJD_Gaza2009_409/816_Pat_001]

(134) „Distanzieren Sie sich entschieden von den Mördern- Feinden der Menschheit.“ [ZJD_Gaza2009_325/816_Kis_001]

Zugleich wird oft unterstellt, Juden würden kognitiv reduziert bzw. unangemessen mit Problemen umgehen, Fehler begehen, kein Verständnis für andere aufbringen und emotional begrenzt sein:

(135) „Ich hasse jegliche Gewalt auch Gewalt gegen Juden. Aber ich wünsche für die Zukunft meiner Kinder eine friedliche Welt. Ihr seid leider nicht daran interessiert, [...]“ [IBD_20.07.2006_ano_008]

(136) „Denken Sie doch mal nach!“ [ZJD_Gaza2009_723/816_Kuh_001]

(137) „was habt ihr nur nicht verstanden als ich schrieb, frieden !!!“ [ZJD_Gaza2009_600/816_ano_001]

(138) „So kann das nicht weitergehen. Sie und der Staat Israel verhalten sich mit einer Frivolität, die ihresgleichen sucht.“ [ZJD_27.07.2006_Rau_001]

Als Begründung werden dann judeophobe Konzeptualisierungen verbalisiert, wie in der E-Mail eines Professors:

(139) „Hintergrund ist wohl die zionistische Idee, ein auserwähltes Volk zu sein.“[ZJD_27.07.2006_Rau_001] 
Die Feststellung oder die als rhetorische Frage formulierte Unterstellung, Juden hätten keine (mit)menschlichen Gefühle, gehört bei diesen Diskreditierungen zu den am häufigsten artikulierten Vorwürfen an den Zentralrat:

(140) „Fassungslos stelle ich fest, daß Ihnen tote Kinder in Gaza und Libanon offenbar gänzlich egal sind.“ [ZJD_27.07.2006_Rau_001]

(141) „Ihr habt keine Achtung vor dem Leben!!!“ [ZJD_Gaza2009_210/816_ano_001]

(142) „Sie HAben keine menschliche gefühle.“ [IBD_02.06.2010_Bos_002]

(143) „Habt ihr so was wie menschliche Gefühle?““[ZJD_22.10.2008_Lie_001]

Auch der Vorwurf, Juden hätten aus der Geschichte nichts gelernt bzw. seien generell in keiner Weise lernfähig, wird signifikant oft artikuliert:

(144) „Haben Sie und Ihresgleichen nichts aus der Geschichte gelernt??“ [ZJD_03.09.2006_Gus_001]

(145) „Dies ist umso bedauerlicher, als daß das jüdische Volk weder aus deren Heilsgeschichte seit den Tagen Abrahams, noch aus den finsteren Tagen des 3. Reiches etwas gelernt hat ... und jetzt [...] dem Treiben im 'Heiligen Land' schweigend zusieht.“ [ZJD_15.04.2002_Fal_001]

Viele Deutsche verstehen sich als Mahner, die sich aus den „Lehren der deutschen Geschichte“ heraus verpflichtet fühlen, nun die Juden zu warnen und zu maßregeln. Diesen wird vor allem Täterschaft oder Mittäterschaft an ,israelischen Verbrechen“ und „zionistischem Staatsterror“ zum Vorwurf gemacht.

(146) „Und diejenigen, die versuchen, Israels Taten zu rechtfertigen, machen sich damit zu dessen Komplizen.“ [ZJD_30.08.2006_Cra_001]

(147) „Betreff: Frau Knobloch - Ihre Kritik gegen uns Deutsche [...] Wir haben aus unseren Fehlern gelernt, ihr begeht diese gerade.“

[ZJD_30.08.2006_Mei_002]

(148) „wir Deutschen haben aus der geschichte gelernt, mir scheint es aber im Zentralrat der Juden da ein Defizit zu geben“ [ZJD_29.08.2006_Bre_001] 
(149) „Wir Deutschen haben bittere Erfahrung mit Auserwählten, Sie müssen das wohl noch lernen.“ [ZJD_27.07.2006_Rau_001]

Viele Verfasser maßen sich zugleich an, Aussagen des Zentralrates und der Botschaft als Lügen, Heuchelei oder Fiktionen zu deklarieren wie in (150) bis (152):

(150) „Offensichtlich sind Sie auf einem Auge blind, weil anders kann man IHRE Scheinheiligkeit nicht interpretieren.“[ZJD_25.10.2006_Sch_001]

(151) „Glauben Sie allen Ernstes, daß Ihnen die Weltgemeinschaft abnimmt, die Entführung israelischer Soldaten sei Anlaß für die Kämpfe der letzten Wochen?““[JD_18.08.2006_Kan_001]

(152) „Angesichts der Tatsachenverdrehung und Verlogenheit mit der Leute wie der Botschafter Stein, Prof.Wolfenssohn, Frau Knobloch und der israelische UN-Botschafter Gillerman die israelischen Kriegsverbrechen in den Palästinensergebieten und im Libanon rechtfertigen, muß sich niemand über einen berechtigten Antizionismus in Deutschland wundern.“[ZJD_04.08.2006_War_001]

Die Forderungen und Ratschläge werden primär über die Semantik der Modalverben müssen, sollen und dürfen zum Ausdruck gebracht. Mittels des Modalverbs müssen lassen sich „Normen und Vorschriften mit hoher Verbindlichkeit“ (Duden 72006: 564) artikulieren:

(153) „Um in dieser Welt als Jude zu bestehen müsst Ihr Euer Tun absolut ändern: [...]“[ZJD_Gaza2009_20/816_ano_001]

(154) „Muss man sich nicht schämen, Frau Knobloch .....?“ [ZJD_05.01.2009_Kil_001]

Zugleich weisen viele Verfasser in diesem Zusammenhang die offizielle Erinnerungskultur in Deutschland zurück: Sie treten als „Mahner“ gegenüber den Juden auf, lehnen aber die mahnende Funktion des Zentralrats entschieden ab (s. hierzu Kap. 9.3.2).

(155) „Herr Spiegel [...] Wann hören Sie endlich mit Ihren Hetzparolen und Schuldzuweisungen gegen die deutsche und jetzt lebende Generation auf?“ [ZJD_24.06.2002_Pin_001, im Anhang: Unterschriften von Freunden und Bekannten] 
„Sie sollten unbedingt Ihre Anspruchsmoral gegenüber nazideutschland, unter denen bis heute Unschuldige Nachgeborene leiden, mal mit der Moral daheim im faschistischen Israel vergleichen. Vielleicht fällt Ihnen was auf. Da haben auch Sie jede Menge Realität aufzuarbeiten. Und ansonsten verbitte ich mir für meine Person jede Bevormundung in Moral und Anspruch aus der Nazizeit.“ [ZJD_01.09.2006_Len_001]

Viele dieser Direktiva mit dem Modalverb sollten ähneln inhaltlich den erzieherischen Direktiva im Imperativ, vgl. (128) bis (130), mit denen die Adressaten aufgefordert werden, sich zu schämen:

$$
\text { „Sie sollten sich schämen.“ [ZJD_Gaza2009_344/816_Bau_001] }
$$

„Sie, die Vertreter Israels, sollten sich schämen.“ [IBD_16.01.2009_Bau_001]

(159) „Sie und Ihr Land sollten sich in Grund und Boden schämen.“ [IBD_19.03.2009_Knö_001]

Die sich selbst zu friedensliebenden, nicht antisemitischen Humanisten erklärenden Verfasser solcher Schreiben konstruieren eine Einstellungs- und Kommunikationsposition, welche die moralische Verurteilung der Adressaten legitimiert: Sie nehmen den Juden gegenüber eine besserwisserische Haltung ein. Aus ihrer verbalisierten Sicht steht es ihnen als moralisch integren Menschen zu, die „dummen Juden“ und/oder „verwerflichen Israelis“ eines Besseren zu belehren und ihnen ihre Schande vor Augen zu führen. Dies geschieht oft durch die Artikulation von Repräsentativa, die an moralische Bewertungen geknüpft sind wie „Gewalt ist nie gut“, „Unterdrückung eines anderen Volkes ist schlecht“ oder „Frieden und Mitmenschlichkeit sind das Wichtigste“. Die Verantwortung für die Einhaltung ethischer Prinzipien bei den Konflikthandlungen wird dabei stets einseitig auf Seiten der Israelis eingefordert. Dass es terroristische und kriegstreiberische Aktivitäten einer anderen Konfliktpartei im Nahostkonflikt gibt und es historisch betrachtet andere Konfliktherde im Nahen Osten gegeben hat, wird geleugnet oder ausgeklammert (s. hierzu das Kap. 7.2.1).

(160) „Herr Präsident, dass Israel um seine Existenz kämpft, ist einfach Unsinn.“ [ZJD_05.04.2002_Stu_001]

(161) „Mit größter Empörung nehme ich wahr, das Ihr zionistischer Staat zigtausende Menschen zu bombardieren und zu vertreiben, die nichts getan 
haben, außer dort zu leben...Machen Sie sich doch bitte klar, daß vor der Ankunft Israels im Nahen Osten Frieden herrschte. Seither ist Krieg. Gibt Ihnen das nicht irgendwie zu denken?“ [ZJD_27.07.2006_Rau_001]

(162) „Israel führte [...] einen brutalen Krieg, [...] gegen die hilflose Zivilbevölkerung Dieser Teufelskreis von Gewalt und Gegengewalt, der nun schon ca. 60 Jahre andauert, muss endlich gestoppt werden.... Diese Spirale von Gewalt - Ungerechtigkeit - Hass muss beendet werden, sonst wird es niemals Frieden geben.“[ZJD_Gaza2009_63/816_Wil_001]

Zum Teil werden diese allgemeingültigen Aussagen auch durch meinungsbekundende Phrasen wie unserer Ansicht nach oder nach meinem Glauben eingeleitet, vgl. (163) und (164).

(163) „In unseren Augen hat jedes Volk ein Recht auf eine Existenz in Würde und Gerechtigkeit.“[ZJD_19.04.2002_Lin_001, mehrere Unterschriften]

(164) „Eines glaube ich aber zu wissen: Freiheit ist unteilbar. Wenn Israel dies nicht anerkennen will, werden die Folgen nicht ausbleiben.“ [IBD_29.03.2004_Sta_001]

Die studierte Verfasserin aus Braunschweig von (165) positioniert sich durch den doppelten Verweis auf ihre mentale und emotionale Normalität gegenüber den Adressaten, denen sie damit diese Eigenschaften implizit abspricht.

(165) „Ich halte mich für einen normal empfindenden und normal intelligenten Menschen mit gesundem Menschenverstand [...] Ich wünsche mir [...] zur Vesöhnung fähige, einsichtige, weise und kluge Staatsmänner und -frauen, die zur richtigen Zeit am richtigen Ort die richtigen Dinge tun.“ [IBD_12.08.2006_Sch_006]

Der Wunsch am Ende der seitenlangen Zuschrift zum Nahostkonflikt mit allen Formen von Anschuldigungen und Diffamierungen gegen Israel impliziert, dass es eben diese klugen Menschen auf Seiten der Israelis nicht gibt.

Hyperbolische Dämonisierungen wie in (166) und referenzielle De-Realisierungen wie in (167) finden sich auch bei Schreibern aus der gebildeten Mitte, wie die folgenden Äußerungen von promovierten Akademikern zeigen:

(166) „Israel ist das mit Abstand größte Übel auf der Welt [...]“ [ZJD_25.10.2006_Sch_001] 
Während explizite und radikale Drohungen vor allem bei rechtsextremistischen Schreibern zu verzeichnen sind, vermitteln die akademischen Schreiber der Mitte ihre Drohungen implizit und reklassifizieren sie meist als Ratschläge, wie folgende Betreffzeilen von E-Mails zeigen:

$$
\begin{aligned}
& \text { „Vernunftruf aus Erwitte“ [IBD_16.01.2009_Bri_001] } \\
& \text { „Besonnenheit ist jetzt gefragt“ [IBD_22.01.2009_Kam_001] }
\end{aligned}
$$

Moralappelle werden auch oft in Form von rhetorischen Fragen formuliert:

(170) „Haben Sie denn keinen Funken von Mitleid in sich?“ [ZJD_30.06.2008_Man_001]

(171) „Kennt Israel keine Scham ? Kein Mitleid ?“ [ZJD_Gaza2009_116/816_Sel_001]

(172) „Gibt es tatsächlich innerhalb der Gemeinschaft der Juden in Deutschland keine weisen, selbstkritischen und menschlich gerecht empfindenden Mitmenschen?“ [ZJD_Gaza2009_502/816_Cri_001]

Kollektiv wird so auf eine implizite Weise das Stereotyp kodiert, Juden seien keine humanistisch geprägten Menschen. Diese „höflichen“ Beleidigungen dienen alle der moralischen Diskreditierung von Juden, denen Mitverantwortung oder sogar Verantwortung für israelische Militäraktionen (die allesamt als unrechtmäßig, unverhältnismäßig und moralisch verwerflich klassifiziert werden) vorgeworfen wird:

(173) „Und diejenigen, die versuchen, Israels Taten zu rechtfertigen, machen sich damit zu dessen Komplizen.“ [ZJD_30.08.2006_Cra_001]

(174) „legen Sie ihre Opferrolle ab, weil sie schon längst mehrfachst Täter sind.“ [ZJD_25.10.2006_Sch_001]

(175) „unschuldige, kleine Kinder, Mütter und Väter durch Angriffe von Israel ums Leben bringen, ich schäme mich für alle Juden !“

[ZJD_05.01.2009_Kil_001] 
(176)

„Sie gehören ganz klar vor ein internationales Gericht wegen Unterstüzung von Kriegsverbrechen Frau Knobloch. Sie und und die Regierungsbande in Israel [...]“ [ZJD_Gaza2009_568/816_Cap_001]

In (173) bis (176) werden die Mitglieder des ZJD zu Tätern stilisiert. Formuliert werden auch allgemeine Warnungen, die sich auf die politische Gesamtlage beziehen. Hyperbolische Behauptungen sind hier dominant:

(177) „Staatsterrorismus von der schlimmsten Art. Auf Euch wartet eine Strafe, eine Erschütterung, was es sein wird, eine Naturkatastrophe oder Zerstörung anderer Art.“ [IBD_13.07.2006_Hel_001]

(178) „der israelische h i t l e r s t a a t gefährdet den weltfrieden!“ [ZJD_Gaza2009_12/816_Mah_001]

Warnungen unterscheiden sich von Drohungen dadurch, dass sie nicht mit Sanktionsankündigungen verbunden sind. Drohungen enthalten immer die Ankündigung möglicher negativer Konsequenzen bei Missachtung des vom Produzenten Eingeforderten, Warnungen prognostizieren lediglich eine Gefahr (wobei weiterführende Konsequenzen und Entwicklungen impliziert werden können):

$$
\text { „Ihre Politik ist brandgefährlich!“ [IBD_07.06.2011_Ört_001] }
$$

Warnen setzt voraus, dass der Produzent über mehr Wissen verfügt oder qualitativ besser unterrichtet ist als der Rezipient und dem Rezipienten unterstellt, die Gefahren nicht zu sehen. Warnungen sind daher stets Ausdruck eines Überlegenheitsgefühls gegenüber den Juden und Israelis. Diese gönnerhafte Haltung schlägt sich auch in Äußerungen wie (180) und (181) nieder:

(180) „Was aber ein menschliches und ethisches Wunder wäre: wenn sich der Zentralrat der Juden in Deutschland von den Kriegsführungsmethoden Israels distanzierte. Dann, erst dann hätte ich wieder Vertrauen zu Ihnenund einen Funken Interesse am Kontakt.“

[ZJD_Gaza2009_11/816_Ges_001]

(181) „Und weil in Palästina immer Juden gelebt haben, sollten dort auch weiterhin immer Juden leben dürfen und neue dazukommen dürfen [...]. Aber einen 'Judenstaat', in dem andere Menschen weniger Rechte haben, finde ich genauso unakzeptabel wie ein Deutschland, in dem Juden, 
Türken oder andere Menschen weniger Rechte haben als Mehrheitsdeutsche.“[ZJD_24.07.2009_Has_001]

Die Verfasser lassen ein ausgeprägt übersteigertes Selbstwertgefühl erkennen, wenn sie die Existenz und die Gestaltung des Staates Israel sowie ihre Beziehung zum Zentralrat der Juden von ihren persönlichen Einstellungen abhängig machen. Das Modalwort dürfen enthält die Bedeutung ‘die Erlaubnis haben'. Der Verfasser der E-Mail in (181) hält es also für angemessen, die Zukunft Israels ${ }^{11}$ gemäß seiner Vorstellung zu gestalten. Es schwingt viel Herablassung und Anmaßung in solchen Äußerungen mit: Die Verfasser sind von der Rechtmäßigkeit ihrer Forderungen und Ermahnungen überzeugt und glauben, kompetent sowie gewichtig genug zu sein, um den ZJD und die IBD maßregeln zu können. Diese Selbsteinschätzung steht jedoch in eklatantem Widerspruch zur Realität: Die meisten der Moralisten und „Nahostexperten“ (die zum Teil auch über das Internet ihre Vorschläge unterbreiten und Kopien ihrer E-Mails an Zeitungen sowie Politiker schicken) sind nach eigenem Bekunden noch nie in dem Land gewesen, dessen Zukunft sie so energisch gestalten wollen. Entsprechend erteilen viele der Schreiber „gute Ratschläge“ und führen sich dem Zentralrat und der Botschaft wie Erwachsene gegenüber kleinen Kindern auf. Typisch hierfür ist z. B. die Anführung von Analogien, die den ,begriffsstutzigen“ Juden bzw. Israelis kognitiv und moralisch auf die Sprünge helfen sollen:

„Ein Tipp vielleicht noch für die verantwortlichen Politiker beider Seiten: Wenn sich zwei Kinder im Kindergarten nicht über eine gerechte Aufteilung (z.B. ein Stück Kuchen) einigen können, dann darf das eine das Streitobjekt teilen und das andere darf sich als erstes seinen Anteil auswählen - selten habe ich eine gerechtere Aufteilung erlebt...“ [ZJD_28.07.2006_Geh_001]

Diese kommunikative Asymmetrie wird oft mittels emotionsbezeichnender Phrasen wie Ich bin sehr traurig, dass ... oder Ich bin enttäuscht von Ihnen eingeleitet; es folgen Moralappelle und Verhaltensmaßregeln.

11 Der Verfasser, ein promovierter Wissenschaftler, der nach eigenen Angaben noch nie in Israel war, den Staat aber als Apartheid- und Kolonialstaat diffamiert, bezieht seine Informationen ausschließlich über Pressemitteilungen. Es ist signifikant, dass auch gebildete und durch Forschungsaktivitäten für die Relevanz von Fakten(überprüfung) sensibilisierte Menschen ihre professionelle Haltung verlieren, wenn sie judeophob eingestellt sind. Dies bestätigt, dass Judenfeindschaft auf einem geschlossenen Weltdeutungssystem basiert, das resistent gegenüber Fakten und rationalen Argumenten ist (vgl. Kap. 9.4.2). 
(183) „ich war sehr traurig, mal wieder zu lesen (auf SPIEGEL Online), dass der ZDJ sich wieder in eine Israel-Kontroverse [= zu Felicia Langers Bundesverdienstkreuz, d. Verf.] eingemischt hat. Sind Sie nicht dazu da, sich um die Juden in Deutschland zu kümmern?““[ZJD_21.07.2009_Has_001]

(184) „Sehr geehrte Damen und Herren, Ich bin sehr enttäuscht und empört [...].“[ZJD_Gaza2009_63/816_Wil_001]

(185) „Israel ist ein ganz anderes Land, warum beschäftigen Sie sich ständig damit? [...] Wie wäre es denn, wenn Sie einen Ableger gründen würden, z.B. 'Deutsch-Israelische Freundschaftsgesellschaft'? Die könnte sich dann öffentlich zu Israel äußern, und der ZDJ würde sich um Belange der deutschen Juden kümmern.“[ZJD_21.07.2009_Has_001]

Auffällig oft werden in diesem Zusammenhang die Verben auffordern, verlangen, appellieren, verbitten und protestieren benutzt. Dies entspricht auch dem Sprachgebrauch im öffentlichen Kommunikationsraum, wie er Verwendung in Internetkommentaren und Pamphleten, wie beim Aufruf von Bremer Boykottbefürwortern, ${ }^{12}$ findet (s. Schwarz-Friesel 2012a).

(187) „Ich möchte Sie auffordern, dies in aller Deutlichkeit ebenfalls zu tun.“ [ZJD_09.04.2002_Sto_001]

(188) „ich protestiere gegen die menschenverachtende Politik des Staates Israel und appelliere an Sie und alle verantwortlichen Politiker Ihres Landes, diesen verbrecherischen Krieg gegen Palästina und Libanon sofort zu beenden. [...] Es ist höchste Zeit, dass der Staat Israel die Wahrheit anerkennt und die Welt und sich selbst nicht noch länger belügt.“ [IBD_19.07.2006_Kno_001]

Implizite Aufforderungen werden als Bitten, Ratschläge, Erwartungen und Ermahnungen formuliert:

12 Der Aufruf ist einsehbar unter: http://www.steinbergrecherche. com/20110500schinaglanchristlichjuedisch.pdf, letzter Zugriff am 02.09.2012. 
(189)

„Sehr geehrte Damen und Herren, liebe Mitmenschen! Ein wenig Toleranz, etwas weniger Beharrlichkeit hilft! Bitte nehmt doch diesen Gedanken mit.“ [IBD_17.01.2009_Hir_001]

(190) „So würde ich auch erwarten, dass sich der Zentralrat der Juden von diesen Gräueltaten in Gaza und auch anderswo distanziert und sich bei den Opfern entschuldigt, im Namen aller hier lebenden Juden und im Namen des Judentums.“ [ZJD_Gaza2009_64/816_Khu_001]

„Ich ermahne ihren Staat Menschlichkeit an den Tag zu legen [...].“ [IBD_17.01.2009_Sch_002]

„Vergleichen Sie mal bitte Herrn Filbinger mit Herrn Sharon. Filbinger war sicher kein Held, der bereit gewesen wäre, sein Leben oder seine Existenz für Widerstand zu riskieren. Ihr Herr Sharon aber ist um einiges schlimmer: In Sabra und Shatila gab es keinen Befehlsnotstand. Herr Sharon war da nämlich der Befehlshaber. Sagen Sie doch mal etwas zu den Mordtaten der Israelis, dann würden Sie vielleicht wieder etwas glaubwürdiger“ [ZJD_16.04.2007_Rau_001]

Dass solche „Ratschläge“ uralte, Juden diffamierende Klischees bedienen und genauso judeophobe Stereotype transportieren wie die rechtsradikalen Beschimpfungen, nur stilistisch auf einer gehobenen Ebene und damit weniger ostentativ, scheint den selbst ernannten Humanisten nicht bewusst zu sein.

Da viele Autoren der E-Mails trotz ihrer judenfeindlichen Argumente als achtbare, integre Menschen betrachtet werden wollen, greifen sie zu verschiedensten Strategien, die die in ihren Texten präsentierten radikalen Inhalte salonfähig machen sollen (s. Kap. 11). Beim „Antisemitismus der Ehrbaren“ (wie Jean Améry 1969 ihn genannt hat) täuschen die verhüllenden und verharmlosenden Verbalisierungen formal die Einhaltung von Höflichkeitskonventionen vor und sind somit als Scheinhöflichkeit einzuordnen (s. hierzu Malicke, in Arbeit). Davon unterscheiden sich zum einen die von Unhöflichkeit geprägten Zuschriften, deren Autoren ohne das Bestreben, ihr Gesicht zu wahren, unkultiviert Beschimpfungen und Beleidigungen ausstoßen und zum anderen die E-Mails, die von aufrichtiger Höflichkeit zeugen. Dem Adressaten kognitive Einsicht, moralische Tugenden und emotionale Empathiefähigkeit abzusprechen, ihn als lernunfähig bzw. -unwillig darzustellen, ist ebenso eine Beleidigung wie ihn als Schwein oder Unmenschen zu beschimpfen, nur weniger vulgär. Menschen die wichtigsten Werte, nämlich Verstand und Gefühl abzuerkennen, kommt ebenso einer Dehumanisierung und Herabstufung gleich. Nur der Stil ist anders, grundsätz- 
lich bleibt die Entwertung, die Diffamierung des kommunikativen Gegenübers. Die Einhaltung von Höflichkeitsfloskeln und der Verzicht auf vulgäre Beschimpfungen vermag diese destruktive Komponente der nur formal ent-radikalisierten Semantik nicht zu vertuschen.

Betrachtet man vergleichend die Texte an den ZJD von 2000 bis 2009 und jene an die IBD von 2003 bis 2011, so fällt insgesamt auf, dass über den Zeitverlauf hinweg der Ton aggressiver, die Argumentation radikaler und die diffamierenden Verbalformen frequenter und damit usueller werden. In den Verbalattacken gegen den Zentralrat kommen seit 2006 mehr Beleidigungen und Drohungen vor. Schreiben an die Israelische Botschaft enthalten zunehmend Beschimpfungen und Verwünschungen. Auch von Schreibern aus der Mitte wird das Existenzrecht des Staates Israel mittlerweile explizit in Frage gestellt. Dabei ist zu konstatieren, dass Schreiben mit aggressiven Sprachhandlungen bis 2006 eher anonym versandt wurden, danach aber vermehrt mit der Angabe von Namen und Anschrift. Zwar wird die radikale Semantik von gebildeten Antisemiten, die sich als AntiAntisemiten erklären, bislang noch größtenteils mit „verbalen Samthandschuhen“, d. h. primär über implizite Formen (also indirekte Sprechakte) artikuliert, doch auch hier offenbart die pseudo-rationale Argumentation eine irrationale Radikalität und emotionale Aggressivität, die sich oft nur wenig vom extremistischen Gedankengut unterscheiden. Die Einhaltung von Höflichkeitskonventionen nimmt ab, die manifesten Kommunikationsformen sprachlicher Gewalt dagegen nehmen zu. Dass im öffentlichen und massenmedialen Kommunikationsraum unwidersprochen und ohne Sanktionsmaßnahmen extrem israelfeindliche Äußerungen sowie Verbal-Antisemitismen benutzt werden, ist hierbei sicher ein wichtiger Einflussfaktor. Hinzu kommt, dass die Nahostberichterstattung seit der Zweiten Intifada und insbesondere anlässlich des Libanon- und Gaza-Konflikts mit hohem Emotionspotenzial und zum Teil monoperspektivischer Tendenz zu Ungunsten Israels verlief. Dass zahlreiche Schreiber immer wieder auf diese Form der Berichterstattung in ihren E-Mails verweisen, ist ein Indikator für die Relaisfunktion der Medien als Vermittlungsinstanz zwischen öffentlicher und individueller Einstellung sowie Meinungsbildung (s. hierzu Beyer/Leuschner 2010 und Schwarz-Friesel 2012c). Die Tabuisierung judenfeindlicher Äußerungen schwindet, die Akzeptanz von Brachialverbalismen in der Öffentlichkeit ${ }^{13}$ steigt.

13 In den letzten Jahren gab es kaum noch eine Talkshow zum Thema Nahost, bei der nicht extrem israel-kritische Gesprächsteilnehmer unkommentiert inadäquate, dämonisierende und de-realisierende Vokabeln wie Apartheidsstaat, Staatsterror etc. sowie NS-Vergleiche artikulieren durften. Gleichsetzungen von Israelis und Juden (vgl. Hart aber fair: Blutige Trümmer in Gaza vom 21.01.2009) werden dabei sogar von den Moderatoren kommuniziert (s. hierzu ausführlich Kap. 7.3). 


\subsection{Lösungsvorschläge für das „Judenproblem“: „Endgültig ausrotten!“ und „Den Staat Israel auflösen“}

„Hoffentlich werden alle Juden mal von der Welt verschwunden sein.“

[IBD_07.07.2006_ano_026]

Viele der Schreiber belassen es nicht bei Beschimpfungen, spezifischen Drohungen und Verwünschungen, sondern artikulieren auch diverse umfassende Lösungsvorschläge für das , jüdische Problem“ oder (projiziert auf Israel) für „den Nahostkonflikt“, „den Judenstaat“, „das Heilige Land“ oder „Palästina“. Bei den meisten dieser „Vorschläge“ wird keine Unterscheidung zwischen allgemeiner jüdischer Existenz und der Existenz des Staates Israel gemacht. Konzeptuell wird also nicht zwischen jüdisch und israelisch differenziert. Bei den meisten gebildeten Verfassern aus der Mitte jedoch wird immer klar abgegrenzt: Scheinbar selbstlegitimiert durch den Verweis, man sei kein Antisemit und habe nichts gegen Juden allgemein, erfolgen die „Lösungen“ immer bezogen auf den Staat Israel, der „,drastisch verändert“, „, abgeschafft“, „, aufgelöst“ oder „internationalisiert" werden solle. Diese Vorschläge werden teils als Appelle, z. B. in Form von Aufforderungen (an Juden, Israel zu verlassen), teils als manifeste Drohungen oder als „Versprechen“ (also implizite Drohungen) formuliert. Als gefühlsausdrückende Expressiva artikuliert, ähneln sie den prophezeienden Verwünschungen und stellen den zukünftigen Untergang aller Juden und/oder des jüdischen Staates Israel als Wunschvorstellung in Aussicht. Gemeinsam ist ihnen, dass sie eine finale Bewältigung der als problematisch erachteten Situation befürworten und an Überlegungen zum prinzipiellen Umgang ${ }^{14}$ mit dem Problem Juden bzw. Israel geknüpft sind.

Bei vielen Schreibern, insbesondere den Vielfachschreibern, die im Laufe der Jahre wiederholt (und zum Teil mehrmals pro Monat) an den ZJD und/oder die IBD Texte schicken, hat es den Anschein, als sei es die wichtigste Aufgabe in ihrem Leben, sich um jüdische bzw. israelische Angelegenheiten zu kümmern. Die obsessive Komponente des Antisemitismus kommt hier stark zum Vorschein (s. Kap.9). Die den generellen Vorschlägen zugrundeliegende alte und tradierte Konzeptualisierung lautet JUDEN SIND DAS GRUNDÜBEL DER WELT. Sprachlich manifestiert sich diese durch Aussagen wie „Ohne Juden wäre die Welt besser

14 Dies unterscheidet sie von den in Kap. 10.1 erörterten Drohungen und Verwünschungen, die partikular ausgerichtet sind. Die Vorschläge richten sich auf das globale Problem und sind an allgemeine Überlegungen bzw. Erlösungsphantasien geknüpft. Allerdings lassen viele der affektgesteuerten Verwünschungen diese Komponente ebenfalls erkennen. 
dran, Juden sind der Abschaum der Menschheit“ oder „Juden sind Unruhestifter/ Störenfriede“. Exemplarisch hierfür sind Beispiele wie (193), (194) und (195):

(193) „Meine Meinung ist folgende: Solange es noch Juden gibt, gibt es kein Frieden auf der Welt“ ${ }^{15}$ [ZJD_08.01.2008_Hei_001]

(194) „Ihr selbst schafft die Grundlage für Antisemitismus in der Welt [...] !!!“ [ZJD_Gaza2009_70/816_ano_001]

(195) „Ich betrachte euch als Abschaum der Menschheit.“ [IBD_15.07.2006_Dei_001]

Es ist die Ultima Ratio des judenfeindlichen Ressentiments, dafür zu sorgen, das von den Juden verkörperte Böse in der Welt zum Wohle der Menschen auszumerzen. Dieser Erlösungsantisemitismus war das ideologische Fundament der nationalsozialistischen „Endlösung“(s. Kap.4.3).

Es lassen sich drei Typen von Lösungsvorschlägen unterscheiden, die regelmäßig von Schreibern verschiedener Herkunft, Bildung und politischer Ausrichtung vorgetragen werden: Radikale Tötungsappelle (die nicht nur auf Israel, sondern immer auf alle Juden weltweit bezogen sind), utopische Umsiedlungsprogramme (die den Nahostkonflikt beenden sollen) und Auflösungsvorschläge, die den Staat Israel in Nahost betreffen. In den radikal-extremistischen Ausrottungsplänen wird Juden „die Pest an den Hals“, „der Untergang“, „die Ausrottung“, „der nukleare Untergang“, „die zweite, diesmal aber bitte richtige Endlösung“ gewünscht. In den extremistischen Vorschlägen wird keine Trennung zwischen Juden und Israelis vorgenommen. In der Konzeptualisierung verschmelzen entsprechend jüdische und israelische Belange:

(196) „Hallo ihr bluttriefenden Judenschweine! Ich bestreite ein Existenzrecht Israels und ein Lebensrecht der jüdischen Pestilenz.“

[IBD_26.04.2009_Kru_001]

Die Vernichtung aller Juden bzw. deren Vertreibung aus der Welt wird als erstrebenswert erachtet:

15 Implizit wird hier über die Aussage, dass erst dann Frieden herrscht, wenn es keine Juden mehr gibt, der Lösungsvorschlag vermittelt, man müsse dafür sorgen, dass es zukünftig keine Juden mehr geben solle. 
(197) „Raus aus deutschland, Raus aus Gaza, Raus aus dieser Welt, Raus aus dem Universum !“ [ZJD_Gaza2009_102/816_ano_001]

(198) „Eines Tages seid ihr ENDLICH ausgerottet.. Die Welt betet dafuer..“ [ZJD_Gaza2009_401/816_Jar_001]

(199) „Ich hoffe so ihr verreckt bald [...] Ich bin ab sofort ANTISEMIT.“ [ZJD_Gaza2009_178/816_ano_001]

(200) „als Futter sollte man euch verarbeiten, damit ihr einmal nützlich seit.“ [IBD_01.08.2006_ano_026]

(201) „verschwindet aus unserem friedlichen leben und führt Kriege auf dem Planeten Mond von mir aus aber lasst uns auf dieser Erde in Ruhe.“ [IBD_12.08.2006_ano_001]

(202) „Euch muss man alle LÖSCHEn!!!““ [IBD_01.05.2010_ano_024]

Viele dieser radikalen Lösungsvorschläge, die den Charakter von irrealen Verfluchungen haben, sind reine Mordphantasien, es finden sich aber auch immer wieder Verweise auf die in der NS-Zeit bereits real praktizierte „Endlösung“ „zum Guten der Menschheit“. Die Vollendung der „Endlösung“ der Nationalsozialisten mittels deren Praktiken wird öfters angeführt, wobei auch auf NS-Lexik zurückgegriffen wird (s. Kap. 6):

(203) „Wird wieder einmal Zeit das richtige Arier den Gashahn aufdrehen!“ [IBD_19.01.2004_ano_001]

(204) „Am Ende wird es wieder eine diemal hoffentlich gruendliche 'Kristallnacht' geben.“[ZJD_Gaza2009_676/816_Pan_001]

(205) „Da wünsche ich mir sehnlichst die Gaskammern wieder [...].“ [IBD_05.08.2008_Dro_001]

(206) „Geht doch in den ofen zurureck wo ihr herkommt!“ [ZJD_30.07.2006_Ger_001]

(207) „und ab in die Gaskammer!“ [ZJD_Gaza2009_677/816_Per_003] 
Für den jüdischen Staat Israel, der das gelebte Judentum in der Welt symbolisiert, werden entsprechende Problemlösungen vorgeschlagen: Der Staat Israel solle „vernichtet“, „zerschlagen“, „radikal verändert“, „aufgelöst“ oder „modifiziert“ werden.

(208) „ich hoffe der Iran hat die BOMBE.“[IBD_01.06.2010_Thi_001]

(209) „Die Israelis sind die Ratten der Welt und sollten allesamt mit Zyankali vergiftet werden, wie man das bei Ratten so macht.“

[IBD_11.04.2007_Dro_001]

In Text (210) werden die Lösungsvorschläge eingebunden in pseudo-rationale Begründungszusammenhänge und affektive Gefühlsbekundungen, die mehrere für den extremen Verbal-Antisemitismus in der Formvariante des Anti-Israelismus charakteristische Komponenten enthalten (s. Kap. 7):

(210) „Hey ihr widerliches israelisches Verbrecherpack, mittlerweile freue ich mich wirklich über jedes israelische Arschloch, das irgendwo umgebracht wird - Nur tote Israelis sind gute Israelis....Hoffentlich verschwindet eure faschistoider Drecksstaat bald von der Landkarte. Da gebe ich gerne noch was dazu ... Ich finde, es wird wirklich an der Zeit eure 'Repräsentaten' hier in diesem Land vielleicht mal ab und an so zu behandeln, wie ihr Ratten es gerade vorgemacht habt.“ [IBD_28.05.2010_ano_018]

Die mentale Geschlossenheit des Weltbildes des Verfassers, in dem die Israelis die aktuellen Repräsentanten des „unmenschlichen, abgrundtief bösen Juden“ sind, wird transparent.

Die weniger drastisch formulierten, aber nicht weniger irrealen Vorschläge bestimmter Schreiber sehen eine Umsiedelung der in Israel lebenden Juden vor und kommen dann bei einem Mehrfachschreiber aus Bochum folgendermaßen zum Ausdruck:

(211) „Mein Vorschlag ist, Israel nach Texas $\mathrm{zu}$ verlegen. Dann gäbe es das Morden nicht mehr. Die Amerikaner mögen die fleißigen Juden, aber das werden Ihre Leute natürlich nicht wollen, diese Lösung wäre zu einfach, sie ziehen das endlose Töten vor.“[IBD_12.06.2006_Sch_001]

(212) „ich hoffe, daß mein Vorschlag, Israel nach Texas/USA zu verlegen, vielleicht doch noch aufgegriffen wird, damit endlich dieser Terror aufhört.“ [IBD_13.07.2006_Sch_004] 
(213) „Mein Vorschlag ist, daß die Regierung Israels ein genügend großes Stück Land in Texas/USA kauft und seine Leute dorthin transferiert.“ [ZJD_27.07.2006_Sch_002]

(214) „Sie hätten die Möglichkeit, das Morden zu beenden, wenn Sie Israel in die USA verlegen würden.“[IBD_11.06.2008_Sch_001]

Diese Utopien werden zum Teil elaboriert, d.h. mit vielen Erklärungen und Begründungen vorgetragen, wie in (215) von dem ausführlich argumentierenden Verfasser aus Erkrath:

(215) „Besteht für die israelische Regierung nicht die Möglichkeit Land in Ägypten zu erwerben, z.B durch Kauf, mieten oder pachten? (Sinai-Halbinsel o.ä.) Ägypten ist nur zu ca. 10 \% besiedelt, der Rest ist Wüste bzw. Steppe. Das es auch ziemlich arm ist, würde Ägypten dadurch zusätzliche Einnahmen erhalten. Die Millionen, die Israel von den USA erhält, könnte doch zum Teil für Landkäufe eingesetzt werden. Auch wäre die EU bestimmt auch zur Hilfe bereit. Aber auch Syrien oder Jordanien könnten vielleicht für zusätzlichen Wohnraum sorgen. Israel müsste dann nicht mehr im Westjordanland oder in Ost-Jerusalem neue Häuser errichten. Stattdessen würde es z.B. für 100 Jahre Land in Ägypten mieten.“ [IBD_16.09.2009_Dap_001]

Umsiedelungspläne und die Auflösung des Staates Israel werden zum Teil zusammen vorgetragen wie in der insgesamt 25 Seiten langen E-Mail an den Zentralrat in (216):

„Ich habe überlegt, wie dieser Konfliktherd für einen potentiellen dritten Weltkrieg ausgeschaltet werden kann, und nach meiner Meinung und reiflichen Überlegung gibt es nur eine dauerhafte Lösung dieses Konfliktes: [...] Nur durch die Auflösung des israelischen Staates kann das Zusammenrotten der Juden unterbunden werden und damit ihre hochgradig aggressiven Tendenzen als vereintes Volk, daß die Erbaggression und Erbfrustration hemmungslos auslebt. Die aus Israel ausgezogenen Juden haben dann die Möglichkeit, sich wieder woanders anzusiedeln.“ [ZJD_30.11.2006_Gel_001]

Zwischen Juden und Israelis wird auch bei den utopischen Vorschlägen in der Regel nicht differenziert: 
(217) „Die Judenfrage muss gelöst werden. Ein Vorschlag wäre für die gläubigen Juden Flüchtlingslager im Iran vorzubereiten.“

[IBD_18.01.2009_ano_001]

Die Vorschläge der gebildeten Verfasser aus der Mitte der Gesellschaft werden als „friedliche, humanistische“ Lösungen für den Nahostkonflikt und den Weltfrieden ausgegeben. Die Problemlösung besteht immer darin, den Staat Israel aufzulösen.

(218) „Am besten für den Frieden auf der Welt und auch für die Juden wäre wenn Israel sich kamplos auflöst und wenn die Juden aus aller Welt die Verbrechen an den Palästinensern durch reichliche Spenden und den Wiederaufbau Plästinas für die Palästinenser wieder gut machen.“ [IBD_30.06.2006_Bec_001]

Dass dabei eine Projektion des judenfeindlichen Ressentiments vollzogen wird, die auf der Konzeptualisierung ISRAEL ALS KOLLEKTIVER JUDE basiert, wird stets vehement geleugnet. In direkter Analogie zur judenfeindlichen Konzeptualisierung, der zufolge Juden das grundlegende Übel, der Störenfried per se in der Welt sind, wird aber Israel entsprechend dämonisiert und ostentativ mit den gleichen Kriterien entwertet (s. ausführlich hierzu Kap. 7):

(219) „Tatsache ist jedoch, daß der Staat Israel im Nahen Osten der Störenfried Nr. 1 ist.“[IBD_24.07.2006_Ehr_001]

(220) „Israel ist der Störenfried in Nahost.“ [IBD_02.08.2006_Ser_002]

(221) „israel ist neben den 'usa' der schlimmste UNRECHTS-STAAT. ... jetzt sind wir endgültig davon überzeugt: Sie und ihr Volk sind Krank, an Geist und Körper!“ [IBD_15.08.2006_Deu_001, fünf Unterschriften]

(222) „Ihr seid das ÜBEL dieser Welt, Krankheiten und Elend über euch und eure Kinder.“ [IBD_31.05.2010_Sch_014]

Wird Israel von radikalen und rechtsextremistischen Verfassern bevorzugt als Krebsgeschwür, Krüppelstaat, Kontergangebilde, Mörderwalze, Missgeburt, Monsterstaat und Terroristennest entwertet, findet die Delegitimierung bei gebildeten und linksliberalen Schreibern ihren Ausdruck in Lexemen wie Unrechtsstaat, Apartheidregime/-staat, zionistischer Anachronismus und Kolonialüberbleibsel. Dabei ist es den Schreibern, die solche Be- bzw. Verurteilungen (meist ohne Ver- 
mischung anti-israelischer Diffamierungen mit judenfeindlichen Kollektivattribuierungen und Synonymsetzungen) aussprechen, sehr wichtig zu betonen, dass dies alles nichts mit Antisemitismus zu tun habe:

(223) „Haben Sie begriffen wo das Übel liegt? Die Welt hat langsam genug von der vom Staat Israel professionell betriebenen Terroristenzucht. [...] Warum werden die Juden immer wieder verfolgt! Das müssen sie sich schon selber fragen. [...] Nennt mich nicht einen Antisemiten, denn das trifft nicht zu.“[IBD_04.07.2006_Str_001]

(224) „Ich bin übrigens der Meinung, dass das Kritisieren vom Verhalten der israelischen Regierung bzw. der Armee nichts mit Antisemitismus oder Rechtsradikalismus zu tun hat.“[ZJD_31.07.2006_Baa_001]

Die meisten der sich selbst als „weltoffen“ charakterisierenden und ,im Geiste der Aufklärung“ argumentierenden Schreiber praktizieren dabei in Bezug auf das Judentum und den Staat Israel eine ausgeprägte Intoleranz, die sich zumeist als Verurteilung und Negierung eines jüdischen Nationalstaates artikuliert:

(225) „Sehr geehrte Damen und Herren jüdischen Glaubens! Es tut mir leid, aber genauso wenig wie Deutschland christlich ist, ist Israel jüdisch. Warum setzen Sie also immerfort Judentum mit Israel gleich [...]?“ [ZJD_Gaza2009_28/816_Pah_001]

Von einem promovierten Unternehmensberater aus Bayern kommt der in einem Nebensatz als „gerecht“ und „humanistisch“ deklarierte Vorschlag: ${ }^{16}$

(226) „Es geht mir nicht um den zionistischen Staat, der gehört friedvoll aufgelöst.“[ZJD_Gaza2009_72/816_Som_001]

Ein Herr aus Wesseling gibt entsprechend mit „freundlichen Grüßen“ den Hinweis:

(227) „Es müsste so schnell wie möglich eine Vollversammlung der UN stattfinden, und die Auflösung diskutiert werden, die Auflösung des Staates Israel.“[IBD_03.03.2008_Moe_001]

Und auch ein promovierter Akademiker aus Offenbach befindet:

16 Dabei belassen es viele der Schreiber nicht nur bei ihren Briefen an den ZJD und die IBD, sondern senden diese Texte sowie diverse Kommentare auch an Presseorgane, Politiker und 
(228)

„Sehr geehrter Herr Botschafter Stein. Die beste Lösung für einen Dauerhaften Frieden im Nahen Osten ist die Auflösung des Staates Israel und Vernichtung aller Waffen.“ [IBD_23.03.2007_Hof_001]

Ein Professor versucht dem Zentralrat (in mehreren E-Mails) klarzumachen, dass der jüdische Charakter des Staates Israel verändert werden müsse: Ein multinationaler Staat, ohne religiöse Präferenzen solle entstehen. Die Entstehungsgeschichte Israels wird dabei mittels einer kruden Analogie zur rassistischen Kolonialpolitik als illegitim und moralisch verwerflich gedeutet:

(229) „Die Entsehung Israels wäre in dieser Form nach 1960 bereits völlig unmöglich gewesen. Im Jahre 1947 wurde die UNO noch von den Kolonialmächten dominiert, die es normal fanden, dass die Weißen weite Teile der nichtweißen Welt beherrschten.“[ZJD_24.07.2009_Has_002]

Auf den Hinweis des Generalsekretärs des ZJD (der dem Professor mehrmals antwortete), Israel sei auch eine Garantie für jüdische Menschen und würde nach dem Holocaust vielen Juden ein Gefühl der Sicherheit geben, erfolgt erneut die de-realisierte Delegitimierung Israels (u.a. durch die irreale Gleichsetzung mit dem ehemaligen Apartheidregime in Südafrika) sowie der belehrende Hinweis, dass „der zionistische Traum eines von Juden beherrschten Nationalstaates aufgegeben werden muss“.

(230) „Die Vorstellung, im Heiligen Land Sicherheit zu finden, mag in der Kolonialzeit Sinn gemacht haben, aber seit der Entkolonialisierung in den 60er Jahren ist klar, dass die Europäer nicht einfach fremdes Land in Afrika oder Asien besitzen dürfen. Israel ist eine Anomalie, die keinen Bestand haben wird. Früher oder später wird eine Lösung [...] gefunden werden müssen.“[ZJD_27.07.2009_Has_003]

Um jede Möglichkeit einer radikalen, israel- oder judenfeindlichen Lesart vor den Adressaten, aber auch vor sich selbst auszuschließen, verweisen solche Verfasser stets auf allgemein anerkannte Vorbilder und deklarieren ihre Lösungen als Resultat ihres humanistisch geprägten Denkens und Fühlens.

(231) „Für Palästina ist die beste Lösung die schon von Albert Einstein und Hanna Arendt befürwortete Einstaatenlösung: Ein säkularer Staat, in

die Bundesregierung, setzen „Lösungsvorschläge“ für den Nahostkonflikt ins Internet und verschicken Rundmails, in denen z. B. zum Boykott israelischer Waren aufgerufen wird. 
dem alle Menschen, unabhängig von ihrer Religion und Ethnizität, dieselben Rechte haben und niemand die anderen $\mathrm{zu}$ beherrschen versucht.“[ZJD_24.07.2009_Has_002]

(232) „dass auch wir in Deutschland uns in erster Linie für Gerechtigkeit einsetzen, und nicht in erster Linie für die Nachkommen der Holocaustopfer (zumal letztere in Israel eine kleine Minderheit sind).“

[ZJD 20.12.2009_Has_005]

Lösungsvorschläge für das ,jüdische Problem“ zu formulieren, gehört seit Jahrhunderten zur Geschichte der Judenfeindseligkeit. Der „älteste Hass der Welt“ hat eine destruktive Dimension, die sich je nach Epoche und Weltbild bzw. Ideologie in Forderungen nach Assimilation oder Aggregation zeigt (s. hierzu die Beispiele in Kap.4; vgl. auch Schwarz-Friesel/Friesel 2012). Im religiösen Bereich bedeutet(e) dies die Aufgabe des jüdischen Glaubens und (Zwangs)Konvertierung, im sozialen und politischen Bereich Ghettoisierung, Diskriminierung, Vertreibung, Ermordung oder Assimilierung. Diese Wunschvorstellungen von NichtJuden gegenüber Juden spiegeln sich bis zum heutigen Tag auch in Äußerungen wie (233) bis (236) wider:

(233) „Der Wille zur wirklichen Integration wäre zu wünschen und ein Schritt nach vorne [...]“ [ZJD_30.05.2007_Gir_001]

$$
\begin{aligned}
& \text { „Passt euch endlich an!“ [ZJD_01.05.2008_Kro_001] } \\
& \text { „Verlasst Palästina!““ [IBD_24.03.2009_Pet_001] }
\end{aligned}
$$

„Verschwindet endlich damit die Menschheit wieder in Frieden leben kann.“[ZJD_Gaza2009_577/816_ano_001]

Die „Lösung“ aus nicht-jüdischer Sicht bedeutet für die Juden dabei immer nur eines: Auslöschung oder Aufgabe ihrer ureigenen Existenz als Juden. ${ }^{17}$ Seit der

17 Dass Juden nicht als Juden existieren sollen, wird auch öffentlich im Internet wiederholt und an verschiedenen Stellen (Foren, Chats, Social Networks etc.) artikuliert. Vgl. hierzu beispielhaft die auf die Homepage von Amazon gestellte Kundenrezension zu dem Buch „Esau's Tears. Modern Anti-Semitism and the Rise of the Jews“von Albert S. Lindemann (2000), in dem explizit die These vertreten wird, dass Juden gehasst werden, weil sie Juden sind und daher ihr Judentum in ihrem eigenen Interesse und im Interesse der nicht-jüdischen Gesellschaft ablegen sollten: „I am firmly convinced that the separation demanded by Jewish religion is the primary cause of anti-semitism, simply because of the implied insult to the 
glühende Judenhasser Hundt-Radowsky vor fast 200 Jahren in seinem Buch Der Judenspiegel zwei prinzipielle Lösungen für das Problem des jüdischen „Ungeziefers" vorgeschlagen hat, haben sich die modernen Varianten trotz der Erfahrung des Holocaust in nichts verändert:

(237) „Am Beßten wäre es jedoch, man reinigte das Land ganz von dem Ungeziefer, und hiezu giebt es gleichfalls zwei Mittel. Entweder, sie durchaus zu vertilgen, oder sie auch, wie Pharao, die Meininger, Würzburger und Frankfurter es gemacht haben, zum Lande hinausjagen [...].“

(Hundt-Radowsky 1819: 146)

Dass Israel expressis verbis als jüdischer Staat auftritt und damit selbstbewusst die jüdische Existenz als legitim deklariert und sein Recht auf Verwirklichung demonstriert, ist für Antisemiten eine ungeheure Provokation: Zwangsläufig wird die Annullierung bzw. „Entjudung“ dieses Staates als notwendig gefordert. Im christlichen Anti-Judaismus forderte man die „ungläubigen“ Juden zur Konvertierung auf, im national-völkischen Antisemitismus die „undeutschen“Juden zur Assimilation und im rassistisch-eliminatorischen Antisemitismus betrieb man für die als „Untermenschen“ klassifizierten Juden die „Endlösung“. Heute wird „im Namen der Gerechtigkeit“ und „im Interesse des Weltfriedens“ die Auflösung Israels gefordert. Die modernen Antisemiten reihen sich mit ihren Vorschlägen ${ }^{18}$ unmittelbar in die tradierte Herangehensweise an das „jüdische Problem“ ein und halten damit die Kontinuität der Judenfeindschaft bis zum heutigen Tag aufrecht.

\footnotetext{
majority culture. If Jews would stop being separate, they would gradually stop being hated, but they would no longer be Jews, either. It's an uncomfortable situation for Jews, dealt with mostly by denying that there is any inherent insult in the traditional refusal to socialize, eat together, and intermarry“ (http://www.amazon.ca/product-reviews/0521593697, letzter Zugriff am 02.09.2012). „The implied insult“ bezieht sich auf das Konzept vom auserwählten Volk Gottes sowie die Beibehaltung jüdischer Sitten und Regeln. Dass die jüdische Existenz als solche als Beleidigung und Herausforderung/Provokation empfunden wird, ist Kern des judenfeindlichen Ressentiments.

18 Vgl. hierzu auch das Video des für die Piratenpartei kandidierenden Dietmar Moews (s. http://www.youtube.com/watch?v=04J08Z5RVfs, veröffentlicht am 04.04.2012), der (nachdem er die üblichen Verschwörungskonstrukte wie z. B. das des „Weltjudentums“ artikuliert) zum Schluss seines Beitrags die Empfehlung ausspricht, dass sich jede Minderheit der Mehrheit anschließen solle.
} 


\section{Fazit}

Die Aggressivität, die in den modernen Texten erkennbar wird, zeigt sich manifest in verbalen Gewalthandlungen, die Juden als Juden beschimpfen, beleidigen, verhöhnen, verunglimpfen und bedrohen. Das Gewalt- und Diskriminierungspotenzial der Sprache artikuliert sich aber auch über Sprechakte, in denen Juden belehrt und ermahnt werden. Sie werden (insbesondere von akademischen Schreibern) als unmündige Personen behandelt, die auf die Ratschläge von Nicht-Juden angewiesen sind. Dadurch wird Juden die Fähigkeit abgesprochen, selbst in der Lage zu sein, kritisch oder intelligent zu urteilen und menschlich zu fühlen. Damit korrelieren Fragen und Feststellungen, die die moralische Integrität und rationale Kompetenz jüdischer Mitbürger in Frage stellen bzw. als nicht gegeben konstatieren. Selbst künftige „Lösungen der Judenfrage“ (oft projiziert auf Israel als „Lösungsvorschläge für den Nahostkonflikt“), die entweder die vollständige Assimilation von Juden oder die Auflösung des Staates Israel fordern, werden (entsprechend dem Sprachgebrauch des national-völkischen Rassismus im 19. Jahrhundert und eliminatorischen Antisemitismus in der NS-Zeit) ohne kritische Reflexion, weder für die Parallelen zum historischen Endlösungsplan noch für die realen Konsequenzen in der Zukunft, vorgebracht. 